\title{
Bifurcation analysis of a composite cantilever beam via 1:3 internal resonance
}

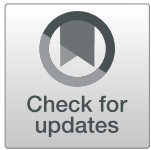

\author{
M. Sayed ${ }^{1 *}$ D, A. A. Mousa ${ }^{2,3}$, D. Y. Alzaharani ${ }^{4}$, I. H. Mustafa ${ }^{5,6}$ and S. I. El-Bendary ${ }^{7}$
}

\author{
*Correspondence: moh_6_11@ \\ yahoo.com; mohamed.abdelkader@ \\ el-eng.menofia.edu.eg \\ 'Department of Engineering \\ Mathematics, Faculty of Electronic \\ Engineering, Menoufia University, \\ Menouf 32952, Egypt \\ Full list of author information is \\ available at the end of the article
}

\begin{abstract}
In this paper, we study a multiple scales perturbation and numerical solution for vibrations analysis and control of a system which simulates the vibrations of a nonlinear composite beam model. System of second order differential equations with nonlinearity due to quadratic and cubic terms, excited by parametric and external excitations, are presented. The controller is implemented to control one frequency at primary and parametric resonance where damage in the mechanical system is probable. Active control is applied to the system. The multiple scales perturbation (MSP) method is implemented to obtain an approximate analytical solution. The stability analysis of the system is obtained by frequency response (FR). Bifurcation analysis is conducted using various control parameters such as natural frequency $\left(\omega_{1}\right)$, detuning parameter $\left(\sigma_{1}\right)$, feedback signal gain $(\beta)$, control signal gain $(\gamma)$, and other parameters. The dynamic behavior of the system is predicted within various ranges of bifurcation parameters. All of the stable steady state (point attractor), stable periodic attractors, unstable steady state, and unstable periodic attractors are determined efficiently using bifurcation analysis. The controller's influence on system behavior is examined numerically. To validate our results, the approximate analytical solution using the MSP method is compared with the numerical solution using the Runge-Kutta (RK) method of order four.
\end{abstract}

Keywords: Active control, Stability, Internal resonance, Bifurcation analysis, Point and periodic attractor

2010 Mathematics Subject Classification: 34D10, 34D20, 34H2O

\section{Springer Open}

\section{Introduction}

An active control system is defined necessarily in terms of that a mass of external power or energy is required. Fanson and Caughey [1] were the first to introduce the Positive Position Feedback (PPF) technique. Vibrations can be abolished via a movement of feedback signals conducted by the mentioned technique. Active constrained layer damping has been effectively implemented as an efficient method to control the vibration of various flexible mechanical structures [2-9]. Eissa and Sayed [10-12] examined the active controller's effect on both spring and simple pendulum at the primary resonance using negative velocity feedback. El-Bassiouny [13], Eissa et al. [14-17], and Jaensch [18] confirmed how active control is functional in vibration attenuation at resonance for different

(c) The Author(s). 2020 Open Access This article is licensed under a Creative Commons Attribution 4.0 International License, which permits use, sharing, adaptation, distribution and reproduction in any medium or format, as long as you give appropriate credit to the original author(s) and the source, provide a link to the Creative Commons licence, and indicate if changes were made. The images or other third party material in this article are included in the article's Creative Commons licence, unless indicated otherwise in a credit line to the material. If material is not included in the article's Creative Commons licence and your intended use is not permitted by statutory regulation or exceeds the permitted use, you will need to obtain permission directly from the copyright holder. To view a copy of this licence, visit http://creativecommons.org/licenses/by/4.0/. 
vibration modes. They approved that the active control method has several advantages with respect to the passive one. Sadek et al. [19] employed piezoelectric-patch-actuators as active control to control the vibration of simply supported flexible plates. An optimal control law was deduced utilizing the maximum principle theory to calculate the voltage of the actuators. Wang et al. [20] used an inverse feed-forward controller method for directing polymer actuator displacement control. Dong et al. [21] investigated the active vibration control method to control piezoelectric smart structures using a system identification technique. Kapuria and Yasin [22] studied the active vibration repression of metal laminated plates through piezoelectric fiber-reinforced composite materials. Warminski et al. [23] studied the experimental and theoretical investigations of vibrations of an autoparametric system composed of two beams with rectangular cross-sections. The experimental response of the system, tuned at 1:4 internal resonance case, is performed for random and harmonic excitations. Warminski et al. [24] investigated the numerical and experimental solutions for various active controllers which applied to a nonlinear mechanical system. El-Ganaini et al. [25] considered the PPF controller to put down the vibration amplitude of a nonlinear dynamic model at primary resonance with $1: 1$ internal resonance. Hamed and Amer [26] studied an active controller for damping out the nonlinear vibration composite beam with parametric excitation force in the case of $1: 2$ internal resonances. Sayed and Kamel [27] examined the effect of a linear controller to control the vibration of each of the saturation control of a linear absorber and the vibrating system. Sayed and Kamel [28] applied an active control method with on $1: 2$ and 1:3 internal resonance to control the vibrations of a nonlinear vibrating system. Large deformation analysis for a cantilever beam with a variable bending stiffness under both static and dynamic loads is investigated by the direct integration scheme [29]. A simple boundary feedback control moment is proposed to stabilize a nonhomogeneous flexible beam with a tip mass [30]. They obtained the exponential stability, spectrum-determined growth condition, and optimal decay rate. Chentouf [31] considered the stabilization problem of a variant of the SCOLE model. He shows that the system uniformly stabilized by the proposed feedback law as soon as the boundary control force and control moment were presented in the feedback. Chentouf [32] studied a model that consists of a nonhomogeneous flexible beam clamped at its left end to a rigid disk and free at the right end, where another rigid body is attached. They treated different physical situations and provided accordingly appropriate feedback control laws. Bağdatlı et al. [33] investigated nonlinear transverse vibrations of a tensioned Euler-Bernoulli beam resting on multiple supports. A perturbation technique was applied to the equations of motion to obtain approximate analytical solutions. He considered 3:1 internal resonance. Hegazy [34] studied the dynamic behavior and chaotic motion of a string-beam coupled system subjected to parametric excitation. He considered and examined the case of $3: 1$ internal resonance between the modes of the beam and the string, in the presence of subharmonic resonance for the beam. Cemil Tunç [35, 36] investigated the asymptotic stability and boundedness of all solutions of certain differential equations of fourth order.

In this paper, the solutions of nonlinear differential equations that represent composite beam vibrations are obtained. We control the vibration of the system via $1: 3$ active control techniques. We applied the MSP technique to solve those nonlinear equations. We extract the corresponding FR equations and present graphically at different system parameters. We apply the RK algorithm to confirm the obtained curves numerically. 
We applied Lyapunov's first method to investigate the stability of the controlled system. To validate our results, we compare approximate solutions using the MSP method and numerical solutions using the RK method of order four. We predict the dynamic behavior of the system at various ranges of bifurcation parameters. We use bifurcation analysis to determine the efficiently stable steady state (point attractor), stable periodic attractor, unstable steady state, and unstable periodic attractors.

\section{Equations of motion}

The modified nonlinear differential equations describe the oscillations of the nonlinear composite beam model $[23,24,26]$ are given by:

$$
\begin{aligned}
& \ddot{u}+2 \mu_{1} \omega_{1} \dot{u}+\omega_{1}^{2} u+\alpha_{1} u^{3}-\delta\left(u \dot{u}^{2}+u^{2} \ddot{u}\right)=f_{1} \cos \Omega_{1} t+u f_{2} \cos \Omega_{2} t+\gamma v^{3}, \\
& \ddot{v}+2 \mu_{2} \omega_{2} \dot{v}+\omega_{2}^{2} v+\alpha_{2} v^{3}=\beta u v^{2},
\end{aligned}
$$

with initial conditions $u(0)=0.02, \dot{u}(0)=0, v(0)=0.2, \dot{v}(0)=0$, where $u$ denotes the composite beam system displacement and $v$ denotes the controller displacement, and the derivatives with respect to the time $t$ are denoted by dots. $\mu_{1}$ and $\mu_{2}$ are the parameters of the viscous damping coefficients, $\omega_{1}$ is the natural frequency accompanied by the composite beam, and $\omega_{2}$ is the natural frequencies associated with controller modes, $\Omega_{1}$ and $\Omega_{2}$ are the excitation frequencies, $f_{1}$ and $f_{2}$ are the excitation forces amplitudes, and $\gamma$ and $\beta$ are the control and feedback signal gains. $\delta, \alpha_{1}$ are the main system nonlinear stiffness coefficient which may be due to material of the beam nonhomogeneity, $\alpha_{2}$ nonlinear stiffness coefficient of the controller. The nonlinear parameters, linear viscous damping, and excitation forces are as follows:

$$
\mu_{n}=\varepsilon \hat{\mu}_{n}, \alpha_{n}=\varepsilon \hat{\alpha}_{n}, \delta=\varepsilon \hat{\delta}, \beta=\varepsilon \hat{\beta}, \gamma=\varepsilon \hat{\gamma}, f_{n}=\varepsilon \hat{f}_{n}, \quad n=1,2,
$$

where the parameter $\varepsilon$ has a low value denoted as perturbation parameter and $0<\varepsilon \ll$ 1. Substituting Eq. (3) into Eqs. (1) and (2), we can obtain:

$$
\begin{aligned}
& \ddot{u}+2 \varepsilon \hat{\mu}_{1} \omega_{1} \dot{u}+\omega_{1}^{2} u+\varepsilon \hat{\alpha}_{1} u^{3}-\varepsilon \hat{\delta}\left(u \dot{u}^{2}+u^{2} \ddot{u}\right)=\varepsilon\left(\hat{f}_{1} \cos \Omega_{1} t+u \hat{f}_{2} \cos \Omega_{2} t\right)+\varepsilon \hat{\gamma} v^{3}, \\
& \ddot{v}+2 \varepsilon \hat{\mu}_{2} \omega_{2} \dot{v}+\omega_{2}^{2} v+\varepsilon \hat{\alpha}_{2} v^{3}=\varepsilon \hat{\beta} u v^{2} .
\end{aligned}
$$

The parameters $\hat{\alpha}_{1}, \hat{\alpha}_{2}, \hat{\mu}_{1}, \hat{\mu}_{2}, \hat{\delta}, \hat{\beta}, \hat{\gamma}, \hat{f}_{1}$, and $\hat{f}_{2}$ are of the order 1 . This means that all these parameters appear when comparing the coefficients of the parameter $\varepsilon$.

\section{Existence and uniqueness theorem}

The system of ODEs (1) and (2) with initial conditions $u(0)=0.02$, $u(0)=0, v(0)=0.2, \dot{v}(0)=0$, can be transformed to a system of first order differential equations IVP in the following:

$$
\begin{aligned}
\text { put } u(t) & =x_{1}(t)=x_{1} \text {, then } \dot{x}_{1}(t)=x_{2}(t)=x_{2} \\
\dot{x}_{2}(t) & =\ddot{u}(t)=\left(f_{1} \cos \Omega_{1} t+x_{1} f_{2} \cos \Omega_{2} t+\gamma x_{3}^{3}-2 \mu_{1} \omega_{1} x_{2}-\omega_{1}^{2} x_{1}-\alpha_{1} x_{1}^{3}+\delta x_{1} x_{2}^{2}\right) /\left(1-\delta x_{1}^{2}\right), \\
\text { put } v(t) & =x_{3}(t)=x_{3} \text {, then } \dot{x}_{3}(t)=x_{4}(t)=x_{4} \\
\dot{x}_{4}(t) & =\ddot{v}(t)=\beta x_{1} x_{3}^{2}-2 \mu_{2} \omega_{2} x_{4}-\omega_{2}^{2} x_{3}-\alpha_{2} x_{3}^{3}
\end{aligned}
$$

and the initial conditions become $x_{1}(0)=0.02, x_{2}(0)=0, x_{3}(0)=0.2, x_{4}(0)=0$. 
Here, we concentrate on the solution of the first order IVP

$$
\begin{aligned}
& \dot{x}_{n}=f\left(t, x_{n}\right) x_{n}(0)=x_{0 n} \quad n=1,2,3,4(*) \\
& \dot{x}_{1}(t)=x_{2}(t) \\
& \dot{x}_{2}(t)=\left(f_{1} \cos \Omega_{1} t+x_{1} f_{2} \quad \cos \Omega_{2} t+\gamma x_{3}^{3}-2 \mu_{1} \omega_{1} x_{2}-\omega_{1}^{2} x_{1}-\alpha_{1} x_{1}^{3}+\delta x_{1} x_{2}^{2}\right) /\left(1-\delta x_{1}^{2}\right) \\
& \dot{x}_{3}(t)=x_{4}(t) \\
& \dot{x}_{4}(t)=\beta x_{1} x_{3}^{2}-2 \mu_{2} \omega_{2} x_{4}-\omega_{2}^{2} x_{3}-\alpha_{2} x_{3}^{3}
\end{aligned}
$$

Theorem 1 [37]. (Existence theorem) Suppose that $f\left(t, x_{n}\right)$ is continuous function in some region $R=\left\{\left(t, x_{n}\right):|t-0| \leq a,\left|x_{n}-x_{0 n}\right| \leq b\right\},(a, b>0)$. Since $f$ is continuous in a closed and bounded domain, it is necessarily bounded in $R$, i.e., there exists $K>0$ such that $\left|f\left(t, x_{n}\right)\right| \leq K \quad \forall\left(t, x_{n}\right) \in R$. Then, the IVP $\left(^{*}\right)$ has at least one solution $x_{n}=x_{n}(t)$ defined in the interval $|t-0| \leq \alpha$ where $\alpha=\min \left(a, \frac{b}{K}\right)$ (note that the solution exists possibly in a smaller interval).

Theorem 2 [37]. (Uniqueness theorem) Suppose that $f$ and $\frac{\partial f}{\partial x_{n}}$ are continuous function in $R$ (defined in the existence theorem). Hence, both the $f$ and $\frac{\partial f}{\partial x_{n}}$ are bounded in $R$, i.e., (a) $\left|f\left(t, x_{n}\right)\right| \leq K$ and (b) $\frac{\partial f}{\partial x_{n}} \leq L \quad \forall\left(t, x_{n}\right) \in R$.

Then, the IVP (*) has at most one solution $x_{n}=x_{n}(t)$ defined in the interval $|t-0| \leq \alpha$ where $\alpha=\min \left(a, \frac{b}{K}\right)$. Combining with existence theorem, the IVP $\left(^{*}\right)$ has a unique solution $x_{n}=x_{n}(t)$ defined in the interval $|t-0| \leq \alpha$. By using Theorems (1) and (2), both the functions $f\left(t, x_{n}\right)$ and its partial derivative are defined and continuous at all points $\{($ $\left.\left.x_{1}, x_{2}, x_{3}, x_{4}\right): x_{1} \neq \pm \sqrt{\frac{1}{\delta}}\right\}$. The theorem guarantees that a solution to the IVP exists in some open interval and that this solution is unique.

\section{Mathematical analysis}

A first-order approximate solution of Eqs. (4) and (5) is conducted using the method of MSP [38, 39].

$$
\begin{aligned}
& u(t ; \varepsilon)=u_{0}\left(T_{0}, T_{1}\right)+\varepsilon u_{1}\left(T_{0}, T_{1}\right), \\
& v(t ; \varepsilon)=v_{0}\left(T_{0}, T_{1}\right)+\varepsilon v_{1}\left(T_{0}, T_{1}\right),
\end{aligned}
$$

where $T_{n}=\varepsilon^{n} t$ is the fast time scales for $n=0$ and slow time scales for $n=1$. In terms of $T_{0}, T_{1}$, the time derivatives are converted to the $D$ operator according to:

$$
\frac{d}{d t}=D_{0}+\varepsilon D_{1}, \quad \frac{d^{2}}{d t^{2}}=D_{0}^{2}+2 \varepsilon D_{0} D_{1},
$$

where $D_{n}=\frac{\partial}{\partial T_{n}}$. After substituting Eqs. (6) and (7) and (8) into Eqs. (4) and (5) and comparing the coefficients of similar powers of the parameter $\varepsilon$ in both sides, we obtain the following:

Order $\left(\varepsilon^{0}\right)$ :

$$
\begin{aligned}
& \left(D_{0}^{2}+\omega_{1}^{2}\right) u_{0}=0, \\
& \left(D_{0}^{2}+\omega_{2}^{2}\right) v_{0}=0 .
\end{aligned}
$$

\section{Order $(\varepsilon)$ :}




$$
\begin{aligned}
& \left(D_{0}^{2}+\omega_{1}^{2}\right) u_{1}=-2 D_{0} D_{1} u_{0}-2 \hat{\mu}_{1} \omega_{1} D_{0} u_{0}-\hat{\alpha}_{1} u_{0}^{3}+\hat{\delta} u_{0}\left(D_{0} u_{0}\right)^{2}+\hat{\delta} u_{0}^{2} D_{0}^{2} u_{0} \\
& \quad+\hat{f}_{1} \cos \Omega_{1} T_{0}+u_{0} \hat{f}_{2} \cos \Omega_{2} T_{0}+\hat{\gamma} v_{0}^{3} \\
& \left(D_{0}^{2}+\omega_{2}^{2}\right) v_{1}=-2 D_{0} D_{1} v_{0}-2 \hat{\mu}_{2} \omega_{2} D_{0} v_{0}-\hat{\alpha}_{2} v_{0}^{3}+\hat{\beta} u_{0} v_{0}^{2} .
\end{aligned}
$$

The solutions of Eqs. (9) and (10) are represented in the complex form:

$$
\begin{aligned}
& u_{0}=A_{1} e^{i \omega_{1} T_{0}}+\bar{A}_{1} e^{-i \omega_{1} T_{0}}, \\
& v_{0}=A_{2} e^{i \omega_{2} T_{0}}+\bar{A}_{2} e^{-i \omega_{2} T_{0}},
\end{aligned}
$$

where $A_{1}$ and $A_{2}$ are complex function with $T_{1}$ as a complex argument, which assigned by removing of secular terms and small-divisor terms from the 1st order of approximation. Therefore, we investigate the case where $\Omega_{1} \cong \omega_{1}, \Omega_{2} \cong 2 \omega_{1}$ and $\omega_{1} \cong 3 \omega_{2}$. To examine quantitatively the closeness of the resonances, the detuning parameters $\sigma_{1}, \sigma_{2}$, and $\sigma_{3}$ are represented by $\Omega_{1}=\omega_{1}+\varepsilon \sigma_{1}, \Omega_{2}=2 \omega_{1}+\varepsilon \sigma_{2}$ and $\omega_{1}=3 \omega_{2}+\varepsilon \sigma_{3}$. Substituting Eqs. (13) and (14) into Eqs. (11) and (12), we get:

$$
\begin{aligned}
\left(D_{0}^{2}+\omega_{1}^{2}\right) u_{1} & =-2 i \omega_{1} D_{1} A_{1} e^{i \omega_{1} T_{0}}+2 i \omega_{1} D_{1} \bar{A}_{1} e^{-i \omega_{1} T_{0}}-2 i \hat{\mu}_{1} \omega_{1}^{2} A_{1} e^{i \omega_{1} T_{0}} \\
& +2 i \hat{\mu}_{1} \omega_{1}^{2} \bar{A}_{1} e^{-i \omega_{1} T_{0}}-\hat{\alpha}_{1} A_{1}^{3} e^{3 i \omega_{1} T_{0}}-3 \hat{\alpha}_{1} A_{1}^{2} \bar{A}_{1} e^{i \omega_{1} T_{0}}-3 \hat{\alpha}_{1} A_{1} \bar{A}_{1}^{2} e^{-i \omega_{1} T_{0}} \\
& -\hat{\alpha}_{1} \bar{A}_{1}^{3} e^{-3 i \omega_{1} T_{0}}-2 \omega_{1}^{2} \hat{\delta} A_{1}^{3} e^{3 i \omega_{1} T_{0}}-2 \omega_{1}^{2} \hat{\delta} A_{1}^{2} \bar{A}_{1} e^{i \omega_{1} T_{0}}-2 \omega_{1}^{2} \hat{\delta} \bar{A}_{1}^{2} A_{1} e^{-i \omega_{1} T_{0}} \\
& -2 \omega_{1}^{2} \hat{\delta} \bar{A}_{1}^{3} e^{-3 i \omega_{1} T_{0}}+\left(\hat{f}_{1} / 2\right) e^{i\left(\omega_{1}+\varepsilon \sigma_{1}\right) T_{0}}+\left(\hat{f}_{1} / 2\right) e^{-i\left(\omega_{1}+\varepsilon \sigma_{1}\right) T_{0}} \\
& +\left(\hat{f}_{2} / 2\right) A_{1} e^{i\left(3 \omega_{1}+\varepsilon \sigma_{2}\right) T_{0}}+\left(\hat{f}_{2} / 2\right) \bar{A}_{1} e^{i\left(\omega_{1}+\varepsilon \sigma_{2}\right) T_{0}} \\
& +\left(\hat{f}_{2} / 2\right) \bar{A}_{1} e^{-i\left(3 \omega_{1}+\varepsilon \sigma_{2}\right) T_{0}}+\left(\hat{f}_{2} / 2\right) A_{1} e^{-i\left(\omega_{1}+\varepsilon \sigma_{2}\right) T_{0}}+\hat{\gamma} A_{2}^{3} e^{i\left(\omega_{1}-\varepsilon \sigma_{3}\right) T_{0}} \\
& +3 \hat{\gamma} A_{2}^{2} \bar{A}_{2} e^{i \omega_{2} T_{0}}+\hat{\gamma} \bar{A}_{2}^{3} e^{-i\left(\omega_{1}-\varepsilon \sigma_{3}\right) T_{0}}+3 \hat{\gamma} \bar{A}_{2}^{2} A_{2} e^{-i \omega_{2} T_{0}} \\
\left(D_{0}^{2}+\omega_{2}^{2}\right) \nu_{1} & =-2 i \omega_{2} D_{1} A_{2} e^{i \omega_{2} T_{0}}+2 i \omega_{2} D_{1} \bar{A}_{2} e^{-i \omega_{2} T_{0}}-2 i \hat{\mu}_{2} \omega_{2}^{2} A_{2} e^{i \omega_{2} T_{0}} \\
& +2 i \hat{\mu}_{2} \omega_{2}^{2} \bar{A}_{2} e^{-i \omega_{2} T_{0}}-\hat{\alpha}_{2} A_{2}^{3} e^{3 i \omega_{2} T_{0}}-3 \hat{\alpha}_{2} A_{2}^{2} \bar{A}_{2} e^{i \omega_{2} T_{0}}-3 \hat{\alpha}_{2} A_{2} \bar{A}_{2}^{2} e^{-i \omega_{2} T_{0}} \\
& -\hat{\alpha}_{2} \bar{A}_{2}^{3} e^{-3 i \omega_{2} T_{0}}+\hat{\beta} A_{1} A_{2}^{2} e^{i\left(\omega_{1}+2 \omega_{2}\right) T_{0}}+2 \hat{\beta} A_{1} A_{2} \bar{A}_{2} e^{i \omega_{1} T_{0}} \\
& +\hat{\beta}_{1} \bar{A}_{2}^{2} e^{i\left(\omega_{2}+\varepsilon \sigma_{3}\right) T_{0}}+\hat{\beta}_{\bar{A}_{1}} A_{2}^{2} e^{-i\left(\omega_{2}+\varepsilon \sigma_{3}\right) T_{0}}+2 \hat{\beta} A_{2} \bar{A}_{1} \bar{A}_{2} e^{-i \omega_{1} T_{0}} \\
& +\hat{\beta} \bar{A}_{1} \bar{A}_{2}^{2} e^{-i\left(\omega_{1}+2 \omega_{2}\right) T_{0}}
\end{aligned}
$$

We get the solvability conditions of the first-order expansion by eliminating the secular terms means the term that has some singularity (an unbounded term arising in time-dependent perturbation theory):

$$
\begin{aligned}
& 2 i \omega_{1} D_{1} A_{1}=-2 i \hat{\mu}_{1} \omega_{1}^{2} A_{1}-3 \hat{\alpha}_{1} A_{1}^{2} \bar{A}_{1}-2 \omega_{1}^{2} \hat{\delta} A_{1}^{2} \bar{A}_{1}+\frac{\hat{f}_{1}}{2} e^{i \sigma_{1} T_{1}}+\frac{\hat{f}_{2}}{2} \bar{A}_{1} e^{i \sigma_{2} T_{1}}+\hat{\gamma} A_{2}^{3} e^{-i \sigma_{3} T_{1}} \\
& 2 i \omega_{2} D_{1} A_{2}=-2 i \hat{\mu}_{2} \omega_{2}^{2} A_{2}-3 \hat{\alpha}_{2} A_{2}^{2} \bar{A}_{2}+\hat{\beta} A_{1} \bar{A}_{2}^{2} e^{i \sigma_{3} T_{1}}
\end{aligned}
$$

By multiplying both sides of Eq. (8) be $2 i \omega_{1}, 2 i \omega_{2}$, we obtain 


$$
\begin{aligned}
& 2 i \omega_{1} \frac{d A_{1}}{d t}=\varepsilon 2 \mathrm{i} \omega_{1} D_{1} A_{1}, \\
& 2 i \omega_{2} \frac{d A_{2}}{d t}=\varepsilon 2 \mathrm{i} \omega_{2} D_{1} A_{2} .
\end{aligned}
$$

Substituting Eqs. (17) and (18) into Eqs. (19) and (20), we obtain

$$
\begin{aligned}
& 2 i \omega_{1} \frac{d A_{1}}{d t}=\varepsilon\left(-2 i \hat{\mu}_{1} \omega_{1}^{2} A_{1}-3 \hat{\alpha}_{1} A_{1}^{2} \bar{A}_{1}-2 \omega_{1}^{2} \hat{\delta} A_{1}^{2} \bar{A}_{1}+\frac{\hat{f}_{1}}{2} e^{i \sigma_{1} T_{1}}+\frac{\hat{f}_{2}}{2} \bar{A}_{1} e^{i \sigma_{2} T_{1}}+\hat{\gamma} A_{2}^{3} e^{-i \sigma_{3} T_{1}}\right), \\
& 2 i \omega_{2} \frac{d A_{2}}{d t}=\varepsilon\left(-2 i \hat{\mu}_{2} \omega_{2}^{2} A_{2}-3 \hat{\alpha}_{2} A_{2}^{2} \bar{A}_{2}+\hat{\beta} A_{1} \bar{A}_{2}^{2} e^{i \sigma_{3} T_{1}}\right) .
\end{aligned}
$$

To analyze Eqs. (21) and (22), we can express $A_{1}$ and $A_{2}$ in polar form as

$$
A_{1}=\frac{1}{2} a_{1} e^{i \gamma_{1}}, A_{2}=\frac{1}{2} a_{2} e^{i \gamma_{2}}
$$

where $a_{1}$ and $a_{2}$ are the steady state amplitudes, and $\gamma_{s}(s=1,2)$ are phases of the motion. Substituting Eq. (23) into Eqs. (21) and (22) and separating the real and imaginary parts, we get the following:

$$
\begin{aligned}
& \dot{a}_{1}=-\mu_{1} \omega_{1} a_{1}+\frac{f_{1}}{2 \omega_{1}} \sin \theta_{1}+\frac{f_{2}}{4 \omega_{1}} a_{1} \sin \theta_{2}+\frac{\gamma}{8 \omega_{1}} a_{2}^{3} \sin \theta_{3}, \\
& a_{1} \dot{\gamma}_{1}=\frac{3 \alpha_{1}}{8 \omega_{1}} a_{1}^{3}+\frac{\delta}{4} \omega_{1} a_{1}^{3}-\frac{f_{1}}{2 \omega_{1}} \cos \theta_{1}-\frac{f_{2}}{4 \omega_{1}} a_{1} \cos \theta_{2}-\frac{\gamma}{8 \omega_{1}} a_{2}^{3} \cos \theta_{3}, \\
& \dot{a}_{2}=-\mu_{2} \omega_{2} a_{2}-\frac{\beta}{8 \omega_{2}} a_{1} a_{2}^{2} \sin \theta_{3}, \\
& a_{2} \dot{\gamma}_{2}=\frac{3 \alpha_{2}}{8 \omega_{2}} a_{2}^{3}-\frac{\beta}{8 \omega_{2}} a_{1} a_{2}^{2} \cos \theta_{3},
\end{aligned}
$$

where

$$
\theta_{1}=\sigma_{1} T_{1}-\gamma_{1}, \theta_{2}=\sigma_{2} T_{1}-2 \gamma_{1}, \theta_{3}=3 \gamma_{2}-\gamma_{1}-\sigma_{3} T_{1} .
$$

Then, it follows from Eq. (28) that

$$
\theta_{1}=\sigma_{1}-\dot{\gamma}_{1} \Rightarrow \dot{\gamma}_{1}=\sigma_{1}-\theta_{1}, \dot{\gamma}_{2}=\frac{1}{3}\left(\theta_{3}+\sigma_{3}+\sigma_{1}-\theta_{1}\right) .
$$

Substituting Eq. (29) into Eqs. (25) and (27), we obtain

$$
\begin{aligned}
& \theta_{1}=\sigma_{1}-\frac{3 \alpha_{1}}{8 \omega_{1}} a_{1}^{2}-\frac{\delta}{4} \omega_{1} a_{1}^{2}+\frac{f_{1}}{2 \omega_{1} a_{1}} \cos \theta_{1}+\frac{f_{2}}{4 \omega_{1}} \cos \theta_{2}+\frac{\gamma}{8 \omega_{1} a_{1}} a_{2}^{3} \cos \theta_{3}, \\
& \theta_{3}=-\sigma_{3}-\frac{3 \alpha_{1}}{8 \omega_{1}} a_{1}^{2}-\frac{\delta}{4} \omega_{1} a_{1}^{2}+\frac{f_{1}}{2 \omega_{1} a_{1}} \cos \theta_{1}+\frac{f_{2}}{4 \omega_{1}} \cos \theta_{2}+\frac{\gamma}{8 \omega_{1} a_{1}} a_{2}^{3} \cos \theta_{3} \\
& +\frac{9 \alpha_{2}}{8 \omega_{2}} a_{2}^{2}-\frac{3 \beta}{8 \omega_{2}} a_{1} a_{2} \cos \theta_{3} .
\end{aligned}
$$

From Eqs. (24), (26), (30), and (31), the autonomous system of equations is:

$$
\dot{a}_{1}=-\mu_{1} \omega_{1} a_{1}+\frac{f_{1}}{2 \omega_{1}} \sin \theta_{1}+\frac{f_{2}}{4 \omega_{1}} a_{1} \sin \theta_{2}+\frac{\gamma}{8 \omega_{1}} a_{2}^{3} \sin \theta_{3},
$$




$$
\begin{aligned}
& \dot{\theta}_{1}=\sigma_{1}-\frac{3 \alpha_{1}}{8 \omega_{1}} a_{1}^{2}-\frac{\delta}{4} \omega_{1} a_{1}^{2}+\frac{f_{1}}{2 \omega_{1} a_{1}} \cos \theta_{1}+\frac{f_{2}}{4 \omega_{1}} \cos \theta_{2}+\frac{\gamma}{8 \omega_{1} a_{1}} a_{2}^{3} \cos \theta_{3} \\
& \dot{a}_{2}=-\mu_{2} \omega_{2} a_{2}-\frac{\beta}{8 \omega_{2}} a_{1} a_{2}^{2} \sin \theta_{3} \\
& \dot{\theta}_{3}=-\sigma_{3}-\frac{3 \alpha_{1}}{8 \omega_{1}} a_{1}^{2}-\frac{\delta}{4} \omega_{1} a_{1}^{2}+\frac{f_{1}}{2 \omega_{1} a_{1}} \cos \theta_{1}+\frac{f_{2}}{4 \omega_{1}} \cos \theta_{2}+\frac{\gamma}{8 \omega_{1} a_{1}} a_{2}^{3} \cos \theta_{3}+\frac{9 \alpha_{2}}{8 \omega_{2}} a_{2}^{2}-\frac{3 \beta}{8 \omega_{2}} a_{1} a_{2} \cos \theta_{3}
\end{aligned}
$$

\section{Steady state solution}

In this work, the steady state motions are examined as follows:

$$
\dot{a}_{1}=\dot{a}_{2}=\dot{\theta}_{1}=\dot{\theta}_{2}=\dot{\theta}_{3}=0 \text {. }
$$

Then, it follows from Eq. (29) that

$$
\dot{\gamma}_{1}=\sigma_{1}, \dot{\gamma}_{2}=\frac{1}{3}\left(\sigma_{1}+\sigma_{3}\right) \text {. }
$$

Then, the steady state solutions of Eqs. (32)-(35) are as follows:

$$
\begin{aligned}
& \mu_{1} \omega_{1} a_{1}-\frac{f_{1}}{2 \omega_{1}} \sin \theta_{1}-\frac{f_{2}}{4 \omega_{1}} a_{1} \sin \theta_{2}-\frac{\gamma}{8 \omega_{1}} a_{2}^{3} \sin \theta_{3}=0 \\
& \sigma_{1}-\frac{3 \alpha_{1}}{8 \omega_{1}} a_{1}^{2}-\frac{\delta}{4} \omega_{1} a_{1}^{2}+\frac{f_{1}}{2 \omega_{1} a_{1}} \cos \theta_{1}+\frac{f_{2}}{4 \omega_{1}} \cos \theta_{2}+\frac{\gamma}{8 \omega_{1} a_{1}} a_{2}^{3} \cos \theta_{3}=0 \\
& \mu_{2} \omega_{2} a_{2}+\frac{\beta}{8 \omega_{2}} a_{1} a_{2}^{2} \sin \theta_{3}=0 \\
& \sigma_{3}+\frac{3 \alpha_{1}}{8 \omega_{1}} a_{1}^{2}+\frac{\delta}{4} \omega_{1} a_{1}^{2}-\frac{f_{1}}{2 \omega_{1} a_{1}} \cos \theta_{1}-\frac{f_{2}}{4 \omega_{1}} \cos \theta_{2}-\frac{\gamma}{8 \omega_{1} a_{1}} a_{2}^{3} \cos \theta_{3}-\frac{9 \alpha_{2}}{8 \omega_{2}} a_{2}^{2}+\frac{3 \beta}{8 \omega_{2}} a_{1} a_{2} \cos \theta_{3}=0 .
\end{aligned}
$$

In addition to a trivial one, two possible cases which will be described as follows exist.

(1) $a_{1} \neq 0, a_{2}=0$ (system without control)

(2) $a_{1} \neq 0, a_{2} \neq 0$ (system with control).

Case (1): We consider $a_{1} \neq 0$ and $a_{2}=0$ (system without control); the FR equation is described as follows:

$$
\left(a_{1} \sigma_{1}-\frac{3 \alpha_{1}}{8 \omega_{1}} a_{1}^{3}-\frac{\delta}{4} \omega_{1} a_{1}^{3}\right)^{2}+\mu_{1}^{2} \omega_{1}^{2} a_{1}^{2}-\frac{f_{1}^{2}}{4 \omega_{1}^{2}}-\frac{f_{2}^{2}}{16 \omega_{1}^{2}} a_{1}^{2}-\frac{f_{1} f_{2}}{8 \omega_{1}^{2}} a_{1}=0 .
$$

Case (2): It is considered $a_{1} \neq 0$ and $a_{2} \neq 0$ (system with control); the FR equations are described as follows:

$$
\left(a_{1} \sigma_{1}-\frac{3 \alpha_{1}}{8 \omega_{1}} a_{1}^{3}-\frac{\delta}{4} \omega_{1} a_{1}^{3}\right)^{2}+\mu_{1}^{2} \omega_{1}^{2} a_{1}^{2}-\frac{f_{1}^{2}}{4 \omega_{1}^{2}}-\frac{f_{2}^{2}}{16 \omega_{1}^{2}} a_{1}^{2}-\frac{f_{1} f_{2}}{8 \omega_{1}^{2}} a_{1}-\frac{\gamma^{2}}{64 \omega_{1}^{2}} a_{2}^{6}-\frac{f_{1} \gamma}{8 \omega_{1}^{2}} a_{2}^{3}-\frac{f_{2} \gamma}{16 \omega_{1}^{2}} a_{1} a_{2}^{3}=0
$$

and 


$$
\begin{aligned}
\left(\sigma_{3}+\frac{3 \alpha_{1}}{8 \omega_{1}} a_{1}^{2}+\frac{\delta}{4} \omega_{1} a_{1}^{2}\right. & \left.-\frac{9 \alpha_{2}}{8 \omega_{2}} a_{2}^{2}\right)^{2}+9 \mu_{2}^{2} \omega_{2}^{2}-\frac{9 \beta^{2}}{64 \omega_{2}^{2}} a_{1}^{2} a_{2}^{2}-\frac{f_{1}^{2}}{4 \omega_{1}^{2} a_{1}^{2}}-\frac{f_{2}^{2}}{16 \omega_{1}^{2}} \\
& -\frac{\gamma^{2}}{64 \omega_{1}^{2} a_{1}^{2}} a_{2}^{6}-\frac{f_{1} f_{2}}{4 \omega_{1}^{2} a_{1}}-\frac{f_{1} \gamma}{8 \omega_{2}^{2} a_{1}^{2}} a_{2}^{3}-\frac{f_{2} \gamma}{16 \omega_{2}^{2} a_{1}} a_{2}^{3}=0 .
\end{aligned}
$$

\section{Stability of the system}

To derive the stability criteria, we assume that

$$
a_{1}=a_{10}+a_{11}, a_{2}=a_{20}+a_{21} \text { and } \theta_{n}=\theta_{n 0}+\theta_{n 1} \quad(n=1,2,3),
$$

where $a_{10}, a_{20}$, and $\theta_{n 0}$ satisfy Eqs. (38)-(41) and $a_{11}, a_{21}$, and $\theta_{n 1}$ are assumed to be small compared with $a_{10}, a_{20}$, and $\theta_{n 0}$. Substituting Eq. (45) into Eqs. (32)-(35), using Eqs. (38)-(41) and keeping only the linear terms in $a_{11}, a_{21}$, and $\theta_{n 1}$, we obtain:

$$
\begin{gathered}
\dot{a}_{11}=\left(\frac{f_{2}}{4 \omega_{1}} \sin \left(2 \theta_{10}\right)-\omega_{1} \mu_{1}\right) a_{11}+\left(\frac{f_{1}}{2 \omega_{1}} \cos \theta_{10}+\frac{f_{2}}{2 \omega_{1}} a_{10} \cos \left(2 \theta_{10}\right)\right) \theta_{11} \\
+\left(\frac{3 \gamma}{8 \omega_{1}} a_{20}^{2} \sin \theta_{30}\right) a_{21}+\left(\frac{\gamma}{8 \omega_{1}} a_{20}^{3} \cos \theta_{30}\right) \theta_{31} \\
\dot{\theta}_{11}=\left(\frac{\sigma_{1}}{a_{10}}-\frac{9 \alpha_{1}}{8 \omega_{1}} a_{10}-\frac{3 \delta}{4} \omega_{1} a_{10}+\frac{f_{2}}{4 \omega_{1} a_{10}} \cos \left(2 \theta_{10}\right)\right) a_{11}-\left(\frac{f_{1}}{2 \omega_{1} a_{10}} \sin \theta_{10}+\frac{f_{2}}{2 \omega_{1}} \sin \left(2 \theta_{10}\right)\right) \theta_{11} \\
+\left(\frac{3 \gamma}{8 \omega_{1} a_{10}} a_{20}^{2} \cos \theta_{30}\right) a_{21}-\left(\frac{\gamma}{8 \omega_{1} a_{10}} a_{20}^{3} \sin \theta_{30}\right) \theta_{31}, \\
\dot{a}_{21}=\left(\frac{\beta}{8 \omega_{2}} a_{20}^{2} \sin \theta_{30}\right) a_{11}+\left(-\mu_{2} \omega_{2}+\frac{\beta}{4 \omega_{2}} a_{10} a_{20} \sin \theta_{30}\right) a_{21}+\left(\frac{\beta}{8 \omega_{2}} a_{10} a_{20}^{2} \cos \theta_{30}\right) \theta_{31}, \\
\dot{\theta}_{31}=\left(-\frac{\sigma_{3}}{a_{10}}-\frac{9 \alpha_{1}}{8 \omega_{1}} a_{10}-\frac{3 \delta}{4} \omega_{1} a_{10}+\frac{9 \alpha_{2}}{8 \omega_{2} a_{10}} a_{20}^{2}-\frac{3 \beta}{4 \omega_{2}} a_{20} \cos \theta_{30}+\frac{f_{2}}{4 \omega_{1} a_{10}} \cos \left(2 \theta_{10}\right)\right) a_{11} \\
-\left(\frac{f_{1}}{2 \omega_{1} a_{10}} \sin \theta_{10}+\frac{f_{2}}{2 \omega_{1}} \sin \left(2 \theta_{10}\right)\right) \theta_{11}+\left(\frac{3 \gamma}{8 \omega_{1} a_{10}} a_{20}^{2} \cos \theta_{30}+\frac{9 \alpha_{2}}{4 \omega_{2}} a_{20}\right. \\
\left.-\frac{3 \beta}{8 \omega_{2}} a_{10} \cos \theta_{30}\right) a_{21}+\left(\frac{3 \beta}{8 \omega_{2}} a_{10} a_{20} \sin \theta_{30}-\frac{\gamma}{8 \omega_{1} a_{10}} a_{20}^{3} \sin \theta_{30}\right) \theta_{31} .
\end{gathered}
$$

The system stability is analyzed by assessing the eigenvalues of the Jacobian matrix of the right-hand sides of the system of Eqs. (46)-(49). The equilibrium solution is considered stable when the real parts of the Jacobian matrix eigenvalues are negative. While the relevant solution is unstable if the corresponding real part of any of the eigenvalues is positive, the fixed points stability is investigated according to the following cases:

Case (1): Uncontrolled system, let $a_{21}=\theta_{31}=0$ in Eqs. (46)-(49); then, the above system is written in the matrix form as follows

$$
\left(\begin{array}{l}
\dot{a}_{11} \\
\dot{\theta}_{11}
\end{array}\right)=\left(\begin{array}{ll}
L_{1} & L_{2} \\
L_{3} & L_{4}
\end{array}\right)\left(\begin{array}{l}
a_{11} \\
\theta_{11}
\end{array}\right)
$$

where 


$$
\begin{aligned}
L_{1} & =-\mu_{1} \omega_{1}+\frac{f_{2}}{4 \omega_{1}} \sin \left(2 \theta_{1}\right), L_{2}=\frac{f_{1}}{2 \omega_{1}} \cos \theta_{1}+\frac{f_{2}}{2 \omega_{1}} a_{1} \cos \left(2 \theta_{1}\right), \\
L_{3} & =\frac{\sigma_{1}}{a_{1}}-\frac{9}{8 \omega_{1}} \alpha_{1} a_{1}-\frac{3 \delta}{4} \omega_{1} a_{1}+\frac{f_{2}}{4 \omega_{1} a_{1}} \cos \left(2 \theta_{1}\right), L_{4} \\
& =-\left(\frac{f_{1}}{2 \omega_{1} a_{1}} \sin \theta_{1}+\frac{f_{2}}{2 \omega_{1}} \sin \left(2 \theta_{1}\right)\right) .
\end{aligned}
$$

Then, the characteristic equation is $\left|\begin{array}{cc}L_{1}-\lambda & L_{2} \\ L_{3} & L_{4}-\lambda\end{array}\right|=0$,

i.e., $\lambda^{2}+B \lambda+C=0$, where $B=-\left(L_{1}+L_{4}\right), C=\left(L_{1} L_{4}-L_{2} L_{3}\right)$, and the obtained eigenvalues are as follows:

$$
\lambda=\frac{\left(L_{1}+L_{4}\right) \pm \sqrt{\left(L_{1}+L_{4}\right)^{2}-4\left(L_{1} L_{4}-L_{2} L_{3}\right)}}{2} .
$$

According to the Routh-Hurwitz criterion, the necessary and sufficient conditions of the roots of Eq. (51) have negative real parts, such that

$$
\left(L_{1}+L_{4}\right)<0, L_{1} L_{4}-L_{2} L_{3}>0
$$

Then, the fixed points are stable; otherwise, they are unstable.

Case (2): Controlled system when $a_{11} \neq 0, \theta_{11} \neq 0, a_{21} \neq 0, \theta_{31} \neq 0$ in Eqs. (46)-(49); then, we can write the above system in the matrix form as shown below:

$$
\left(\begin{array}{c}
\dot{a}_{11} \\
\dot{\theta}_{11} \\
\dot{a}_{21} \\
\dot{\theta}_{31}
\end{array}\right)=\left(\begin{array}{cccc}
L_{1} & L_{2} & L_{9} & L_{10} \\
L_{3} & L_{4} & L_{11} & L_{12} \\
L_{5} & L_{6} & L_{13} & L_{14} \\
L_{7} & L_{8} & L_{15} & L_{16}
\end{array}\right)\left(\begin{array}{c}
a_{11} \\
\theta_{11} \\
a_{21} \\
\theta_{31}
\end{array}\right) .
$$

The eigenvalue of the above system is as follows:

$$
\left|\begin{array}{cccc}
L_{1}-\lambda & L_{2} & L_{9} & L_{10} \\
L_{3} & L_{4}-\lambda & L_{11} & L_{12} \\
L_{5} & L_{6} & L_{13}-\lambda & L_{14} \\
L_{7} & L_{8} & L_{15} & L_{16}-\lambda
\end{array}\right|=0,
$$

i.e. $\lambda^{4}+R_{1} \lambda^{3}+R_{2} \lambda^{2}+R_{3} \lambda+R_{4}=0$, (54)

where

$$
\begin{aligned}
& L_{5}=\frac{\beta}{8 \omega_{2}} a_{2}^{2} \sin \theta_{3}, L_{6}=0, \\
& L_{7}=\left(-\frac{\sigma_{3}}{a_{1}}-\frac{9 \alpha_{1}}{8 \omega_{1}} a_{1}-\frac{3 \delta}{4} \omega_{1} a_{1}+\frac{9 \alpha_{2}}{8 \omega_{2} a_{1}} a_{2}^{2}-\frac{3 \beta}{4 \omega_{2}} a_{2} \cos \theta_{3}+\frac{f_{2}}{4 \omega_{1} a_{1}} \cos \left(2 \theta_{1}\right)\right), \\
& L_{8}=-\left(\frac{f_{1}}{2 \omega_{1} a_{1}} \sin \theta_{1}+\frac{f_{2}}{2 \omega_{1}} \sin \left(2 \theta_{1}\right)\right), L_{9}=\frac{3 \gamma}{8 \omega_{1}} a_{2}^{2} \sin \theta_{3}, L_{10}=\frac{\gamma}{8 \omega_{1}} a_{2}^{3} \cos \theta_{3}, \\
& L_{11}=\frac{3 \gamma}{8 \omega_{1} a_{1}} a_{2}^{2} \cos \theta_{3}, \quad L_{12}=-\frac{\gamma}{8 \omega_{1} a_{1}} a_{2}^{3} \sin \theta_{3}, L_{13}=-\mu_{2} \omega_{2}+\frac{\beta}{4 \omega_{2}} a_{1} a_{2} \sin \theta_{3}, \\
& L_{14}=\frac{\beta}{8 \omega_{2}} a_{1} a_{2}^{2} \cos \theta_{3}, \quad L_{15}=\left(\frac{3 \gamma}{8 \omega_{1} a_{1}} a_{2}^{2} \cos \theta_{3}+\frac{9 \alpha_{2}}{4 \omega_{2}} a_{2}-\frac{3 \beta}{8 \omega_{2}} a_{1} \cos \theta_{3}\right),
\end{aligned}
$$




$$
L_{16}=\left(\frac{3 \beta}{8 \omega_{2}} a_{1} a_{2} \sin \theta_{3}-\frac{\gamma}{8 \omega_{1} a_{1}} a_{2}^{3} \sin \theta_{3}\right),
$$

$R_{1}, R_{2}, R_{3}$, and $R_{4}$ are functions of $L_{s}(s=1,2, \ldots, 16)$ which are given above. Based on the Routh-Hurwitz criterion, the necessary and sufficient conditions of the roots of Eq. (54) have negative real parts, such that

$$
R_{1}>0, R_{1} R_{2}-R_{3}>0, R_{3}\left(R_{1} R_{2}-R_{3}\right)-R_{1}^{2} R_{4}>0, R_{4}>0 .
$$

In the following figures, the dashed lines refer to unstable solutions, while the solid lines refer to stable solutions.

\section{Numerical results}

Simulation results are illustrated in graphical forms such as the steady state amplitude of the composite beam system versus time history and detuning parameter $\sigma_{1}$ or the response of the composite beam system and controller at $1: 3$ internal resonance. The different parameters of the composite beam system shown in Fig. 1 are $\mu_{1}=0.001, f_{1}=$ $0.01, f_{2}=0.05, \alpha_{1}=0.002, \omega_{1}=4, \delta=0.0001$. Figure 1 declares that the steady state amplitude of the composite beam system is about 90 times that of the external excitation amplitude $f_{1}$.This is conducted when the controller is idle at the simultaneous primary and principal parametric resonance.

Figure 2 shows that the time history for both composite beam system and controller amplitudes at $\Omega_{1} \cong \omega_{1}, \Omega_{2} \cong 2 \omega_{1}$ at internal resonance $\omega_{1} \cong 3 \omega_{2}$. From Fig. 2 , it is observed that the energy is transmitted from the composite beam to the controller. To measure the effectiveness of the controller, we define $E_{a}$ as flows ( $E_{a}=$ uncontrolled system amplitude/controlled system amplitude). Then, the effectiveness of the controller $E_{a}$ is about 35 . Figures $3,4,5,6$, and 7 indicate numerical results of the frequency response curves of the controlled system. Figure 3 illustrates that the response of the controlled system against $\sigma_{1}$. We note that the minimum response of the beam occurs when $\sigma_{1}=0$, which approves that the controller is able to eliminate the simultaneous

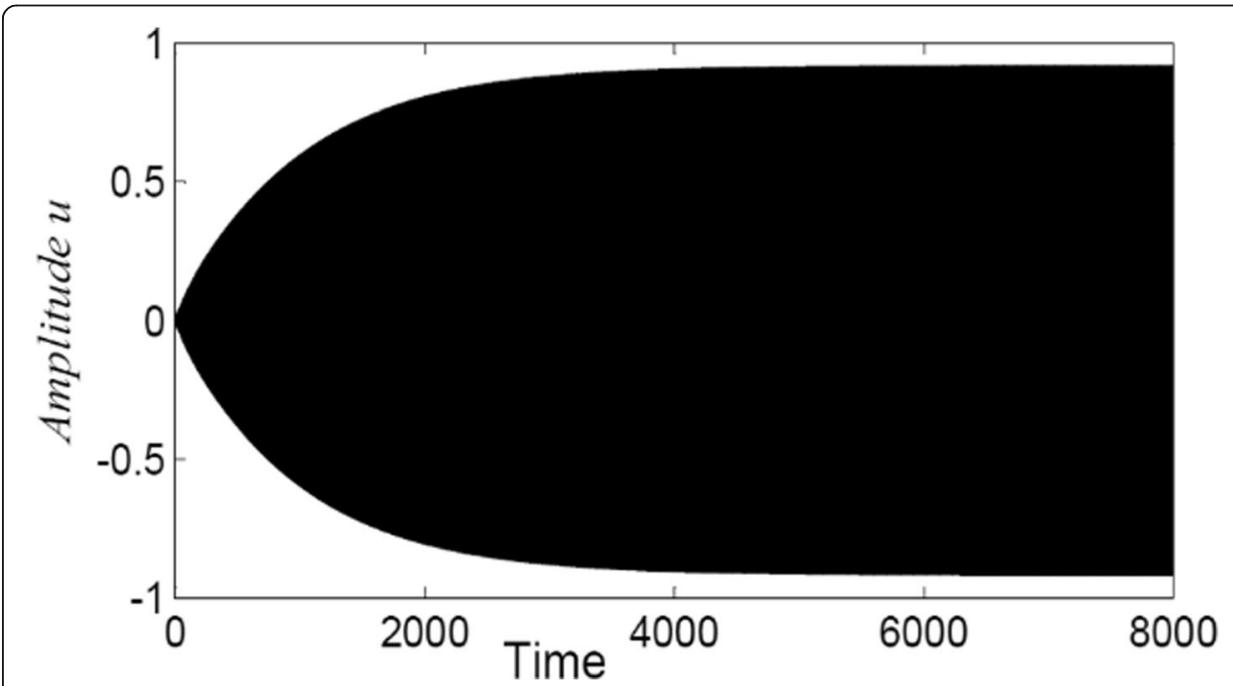

Fig. 1 System behavior without a controller at resonance $\Omega_{1} \cong \omega_{1}, \Omega_{2} \cong 2 \omega_{1}$ for initial conditions $u(0)=.02, \dot{u}(0)=0$ 

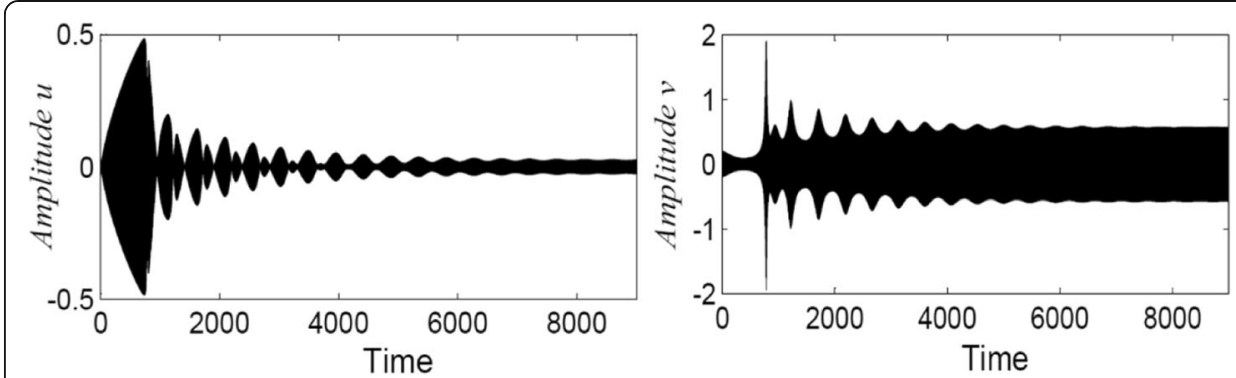

Fig. 2 System behavior with a controller at resonance $\Omega_{1} \cong \omega_{1}, \Omega_{2} \cong 2 \omega_{1}, \omega_{1} \cong 3 \omega_{2}$ for initial conditions $u(0)=0.02, \dot{u}(0)=0, v(0)=0.2, \dot{v}(0)=0 . \mu_{1}=0.001, \mu_{2}=0.00098, f_{1}=0.01, f_{2}=0.05, a_{1}$ $=0.002, a_{2}=0.002, \omega_{1}=4, \delta=0.0001, \beta=1, \gamma=0.2$

primary and parametric resonance vibration effectively at the existence of the 1:3 internal resonance. Figure 4 shows that the influence of $f_{1}$ on the system amplitude response against the detuning parameter $\sigma_{1}$. From these figures, it is noticed that the increase in excitations force $f_{1}$ leads to an increase in the region of instability system. Figure 5 shows the effects of changing the control signal $\gamma$ on the FR curves of both the controller and the beam system. Also, for decreasing $\gamma$, the effective frequency bandwidth of the saturation controller induces lowering the vibration impact of the composite beam. On the other hand, the controller's excessive risk is decreased when the control signal is raised.

Figure 6 demonstrates the effect of feedback signal $\beta$ variation on the FR curves. Form this figure, we can see that decreasing the feedback signal obtained a better vibration lowering influence on the composite beam. Figure 7 indicates that the impacts of the natural frequency on the amplitude response curves against $\sigma_{1}$. Also, it is observed that for increasing the natural frequency, the effective frequency bandwidth of the controller and the better vibration damping influence of the composite beam.

\section{Bifurcation analysis}

Dynamic bifurcation analysis is performed using the XPPAUT 5 software package and AUTO-07p. Furthermore, MATLAB is used for investigating the dynamic behavior where the dynamic results are obtained at specific parameter values defined from AUTO 2007 [40-43]. The bifurcation analysis is performed for most of the controlling parameters such as parametric excitations parameters $\left(f_{2}\right)$, nonlinear control parameter
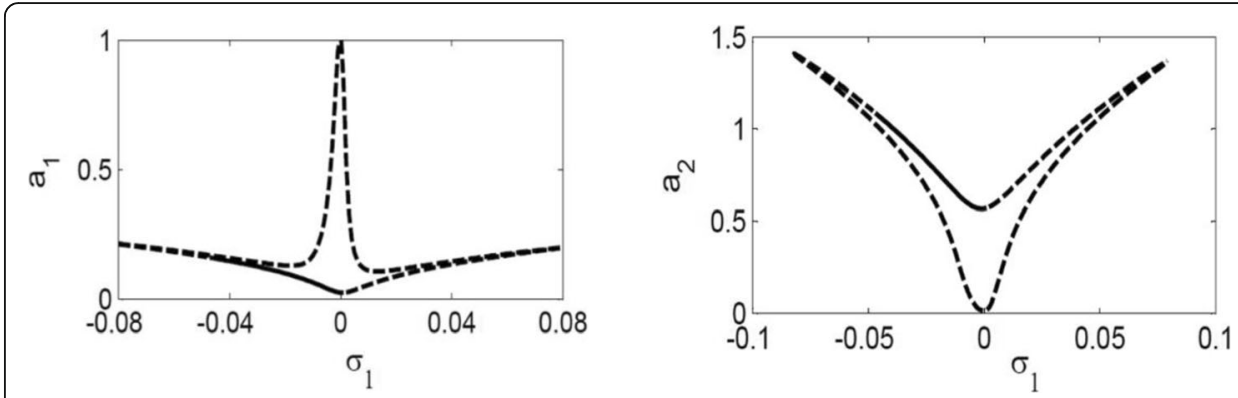

Fig. 3 Effects of the detuning parameter $\sigma_{1}$ for $\mu_{1}=0.001, \mu_{2}=0.00098, f_{1}=0.01, f_{2}=0.05, a_{1}=0.002, a_{2}=$ $0.002, \omega_{1}=4, \delta=0.0001, \beta=1, \gamma=0.2$ 


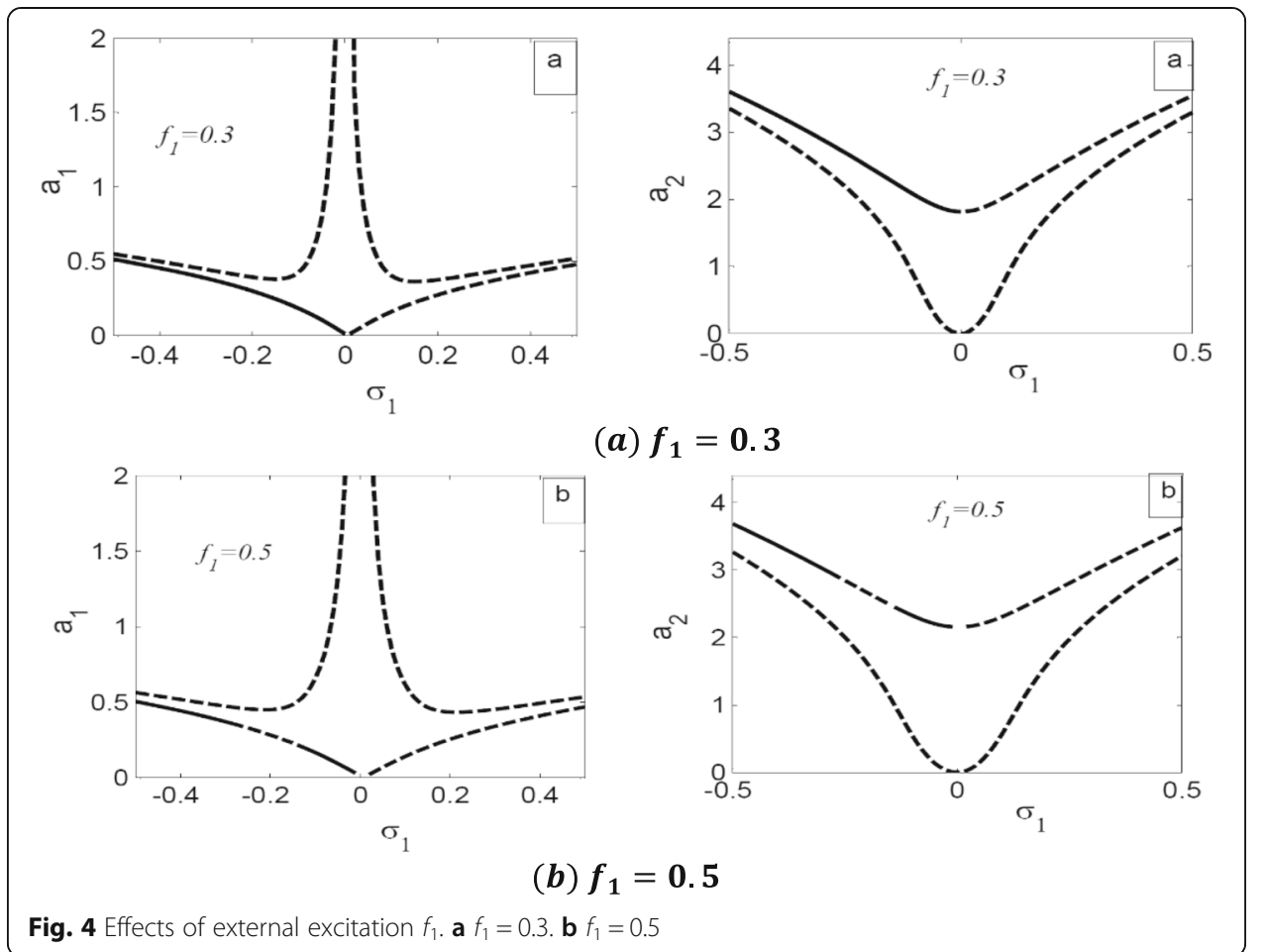

$\left(\alpha_{2}\right)$, detuning parameter $\left(\sigma_{1}\right)$, feedback signal gain $(\beta)$, control signal gain $(\gamma)$, and the natural frequency $\left(\omega_{1}\right)$ as shown below.

\section{Effect of the parametric excitation parameter $\left(f_{2}\right)$}

Figure $8 \mathrm{a}$ and $\mathrm{b}$ show the effect of the parametric excitation bifurcation parameter $\left(f_{2}\right)$ on $a_{1}$ and $a_{2}$, respectively. The parameter values are indicated in Table 1 . There is only Hopf bifurcation (HB) point at $f_{2}=0.06839$, where $a_{1}=0.02473$ and $a_{2}=0.5691$. In the region of $\left(f_{2} \leq 0.06839\right)$, the system is dominated by a stable steady state, where $a_{1}$ is around 0.025 and $a_{2}$ is around 0.57 . In the region of $\left(0.06839 \leq f_{2} \leq 0.09\right)$, the system is characterized by stable periodic attractor, where $a_{1}$ oscillated in the range of $[0.02,0.28]$ while $a_{2}$ oscillated in the range of $[0.4,1.32]$; there is the unstable steady state in the same region $\left(0.06839 \leq f_{2} \leq 0.09\right)$ and unstable periodic attractor in $\left(0.085 \leq f_{2} \leq 0.09\right)$.
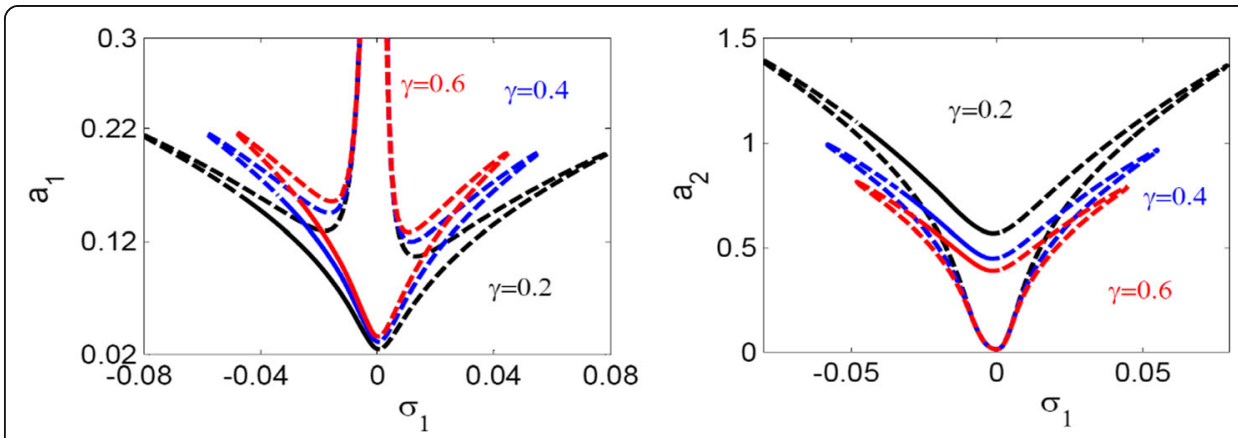

Fig. 5 Effects of control signal gain $\gamma$ 

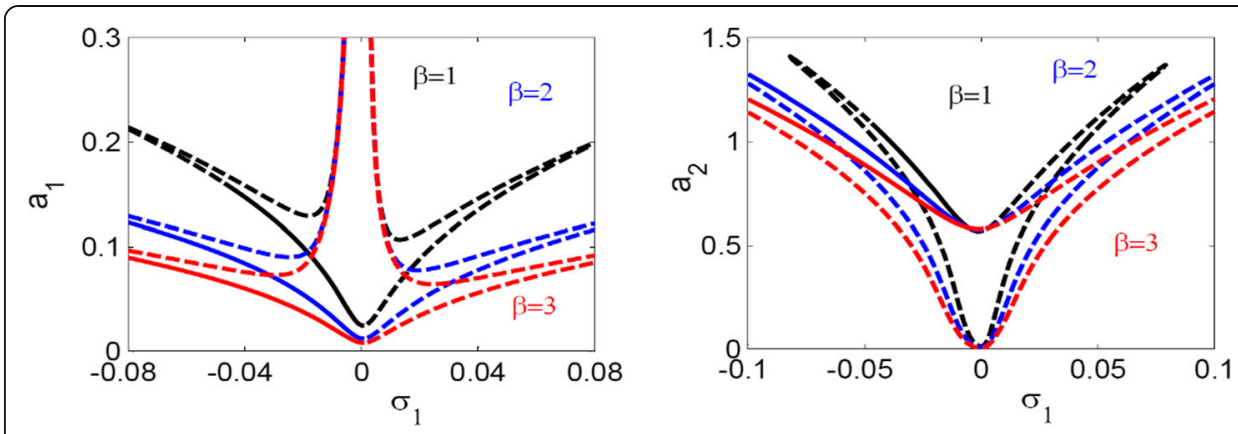

Fig. 6 Effects of feedback signal gain $\beta$

\section{Effect of the nonlinear control parameter $\left(a_{2}\right)$}

Figure $9 \mathrm{a}$ and $\mathrm{b}$ indicate the effects of the nonlinear control parameter $\left(\alpha_{2}\right)$ as a bifurcation parameter on $a_{1}$ and $a_{2}$ at $\gamma=30$ based on the two-parameter continuation technique [44], and the rest of the parameter's values are shown in Table 1. There are two Hopf bifurcation points: the first $\mathrm{Hopf} \mathrm{HB}_{1}$ appears at the two points $\left(\alpha_{2}, a_{1}\right)=(0.478$, $0.2006)$ and $\left(\alpha_{2}, a_{2}\right)=(0.478,0.09285)$, while second Hopf $\mathrm{HB}_{2}$ appears at the two points $\left(\alpha_{2}, a_{1}\right)=(0.1008,0.1447)$ and $\left(\alpha_{2}, a_{2}\right)=(0.1008,0.09622)$. The system behavior can be characterized as follow:

Region $\left(\alpha_{2} \leq 0.1008\right)$ : The bifurcation diagrams obtained in Fig. 9 a, and $b$ show that there exists a point attractor only, if $a_{1}$ changes in the range of $[0.148,0.18]$ while $a_{2}$ changes in the range of $[0.096,0.1]$.

Region $\left(0.1008 \leq \alpha_{2} \leq 0.478\right)$ : The system is characterized by the unstable steady state through this region in the range $\left(0.1008 \leq \alpha_{2} \leq 0.18\right)$; the system is dominated by the stable periodic attractor. Figure $10 \mathrm{a}-\mathrm{c}$ indicate the dynamics of the system at $\alpha_{2}=0.12$ at the corresponding initial conditions. Figure 10 a shows the time course of $a_{1}$, where $a_{1}$ fluctuates in the range of $[0.113,0.185]$ while time traces of $a_{2}$ shows that $a_{2}$ fluctuates in the range $[0.0885,0.1035]$ as shown in Fig. 10b. The phase plane is shown in Fig. 10c confirms the oscillatory attractor which is the only attractor at $\alpha_{2}=0.12$ as shown through the regular limit cycles of $a_{1}$ and $a_{2}$.

Region $\left(0.478 \leq \alpha_{2}\right)$ : The system is dominated by a stable steady state. There is also an unstable periodic attractor. Figure $11 \mathrm{a}$ and $\mathrm{b}$ show time traces of $a_{1}$ and $a_{2}$ at $\alpha_{2}=$ 0.8 at the corresponding initial conditions where $a_{1}$ settles down at $a_{1}=0.27$ while $a_{2}=$
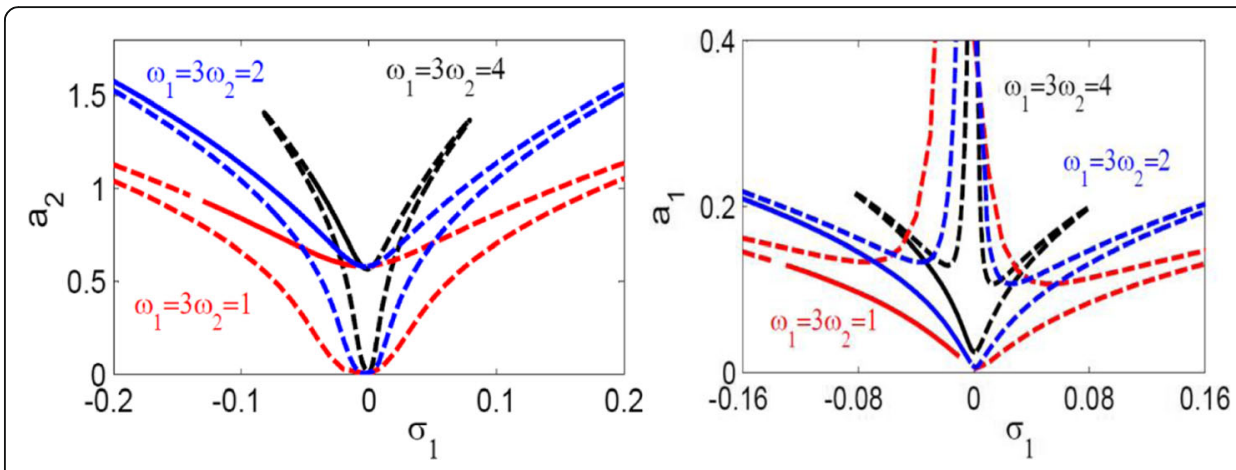

Fig. 7 Effects of the natural frequency $\omega_{1}$ 

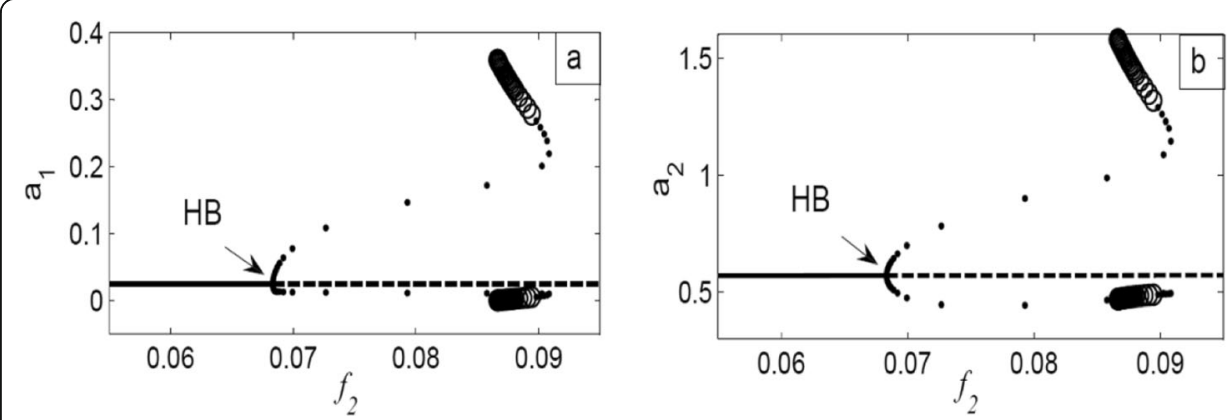

Fig. 8 Bifurcation diagram of $f_{2}$ as the bifurcation parameter. Stable steady state branch, thick black line; unstable steady state branch, thick broken black lines; stable periodic branch, black circles; unstable periodic branch, hollowed/white circles

0.0923. Phase plane (Fig. 11c) confirms the time traces in Fig. 11a, b where the system approached a point attractor.

\section{Effect of the detuning parameter $\left(\sigma_{1}\right)$}

Figure $12 \mathrm{a}$ and $\mathrm{b}$ show the bifurcation analysis using the detuning parameter $\left(\sigma_{1}\right)$ as the bifurcation parameter at $\gamma=50$ based on the two-parameter continuation technique [44], and the rest of the parameter's values are indicated in Table 1. Two Hopf bifurcation points are available: $\mathrm{HB}_{1}$ appears at $\sigma_{1}=5.95 \times 10^{-5}, a_{1}=0.1722$, and $a_{2}=0.08094$, while $\mathrm{HB}_{2}$ is at $\sigma_{1}=-0.001926, a_{1}=0.1875$, and $a_{2}=0.08292$ as shown in Fig. $12 \mathrm{a}$, b.

Region $\left(5.95 \times 10^{-5} \leq \sigma_{1}\right)$ : The system is dominated by the point attractor where there is only a stable steady state.

Region $\left(-0.001926 \leq \sigma_{1} \leq 5.95 \times 10^{-5}\right)$ : In this region, the system is dominated by the only periodic attractor. There is also an unstable steady state. The maximum frequency for $a_{1}$ and $a_{2}$ appears close to $\mathrm{HB}_{1}$ at $\sigma_{1}=0$, where $a_{1}$ oscillates in the range of [0.02, $0.45]$ as shown in Fig. 12a, and $a_{2}$ oscillates in the range of $[0.045,0.135]$ as shown in Fig. 12b. However, this frequency decreases continuously as $\sigma_{1}$ decreases until reaching $\mathrm{HB}_{2}$ as shown in Fig. 12a, b.

Region $\left(\sigma_{1} \leq-0.001926\right)$ : In this region, the system is dominated by the point attractor where a stable steady state is available, where $a_{1}$ changes in the range [0.172, $0.19]$ while $a_{2}$ changes in the range $[0.082,0.09]$ as shown in Fig. 12a, b.

\section{Effect of the feedback signal gain ( $\beta$ )}

Figure 13 shows the effect of feedback signal $\beta$ as the main bifurcation parameter where other parameter values are shown in Table 1 . There is only one HB at $\beta=0.1776$ and $a_{1}=0.1544$, and $a_{2}=0.5116$ as shown in Fig. 13a, b, respectively.

Table 1 Parameter values

\begin{tabular}{llllll}
\hline Parameter & Value & Parameter & Value & Parameter & Value \\
\hline$\gamma$ & 0.2 & $a_{1}$ & 0.002 & $\sigma_{1}$ & 0 \\
$a_{2}$ & 0.002 & $\beta$ & 1 & $\delta$ & 0.0001 \\
$\mu_{1}$ & 0.001 & $f_{1}$ & 0.01 & $\sigma_{3}$ & 0 \\
$\mu_{2}$ & 0.00098 & $f_{2}$ & 0.05 & $\omega_{1}$ & 4 \\
\hline
\end{tabular}




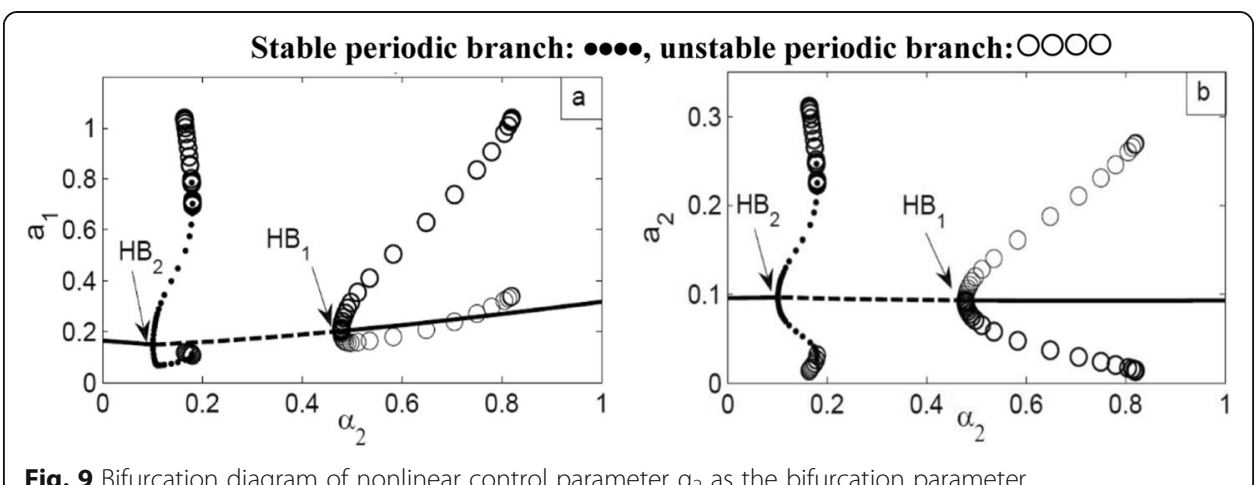

Fig. 9 Bifurcation diagram of nonlinear control parameter $a_{2}$ as the bifurcation parameter

Region $(0.1776 \leq \beta)$ : The system is controlled by only a stable steady state, where $a_{1}$ changes in the range of $\left[\begin{array}{ll}0.1 & 0.1544\end{array}\right]$ (Fig. 13a) while $a_{2}$ changes in the range of [0.1, $0.51]$.

Region $(0.133 \leq \beta \leq 0.1776)$ : In this region, the system is controlled by stable periodic attractor where $a_{1}$ changes in the range of $[0.01,1]$ as shown in Fig. 13a while $a_{2}$ oscillates in the range of $[0.1,1.4]$ as shown in Fig. 13b.

Region $(\beta \leq 0.133)$ : The system has no stable behavior where there are unstable steady state and unstable periodic behavior as shown in Fig. 13a, b.

\section{Effect of the control signal gain $(\gamma)$}

Figure 14 shows the effect of control signal $\gamma$ as the main bifurcation parameter where the rest of the parameter's values are indicated in Table 1 . There is only one HB at $\gamma=$ 47.56 and $a_{1}=0.1691$, and $a_{2}=0.08244$ as shown in Fig. 14a, b, respectively. The behavior of the system can be described as follows:

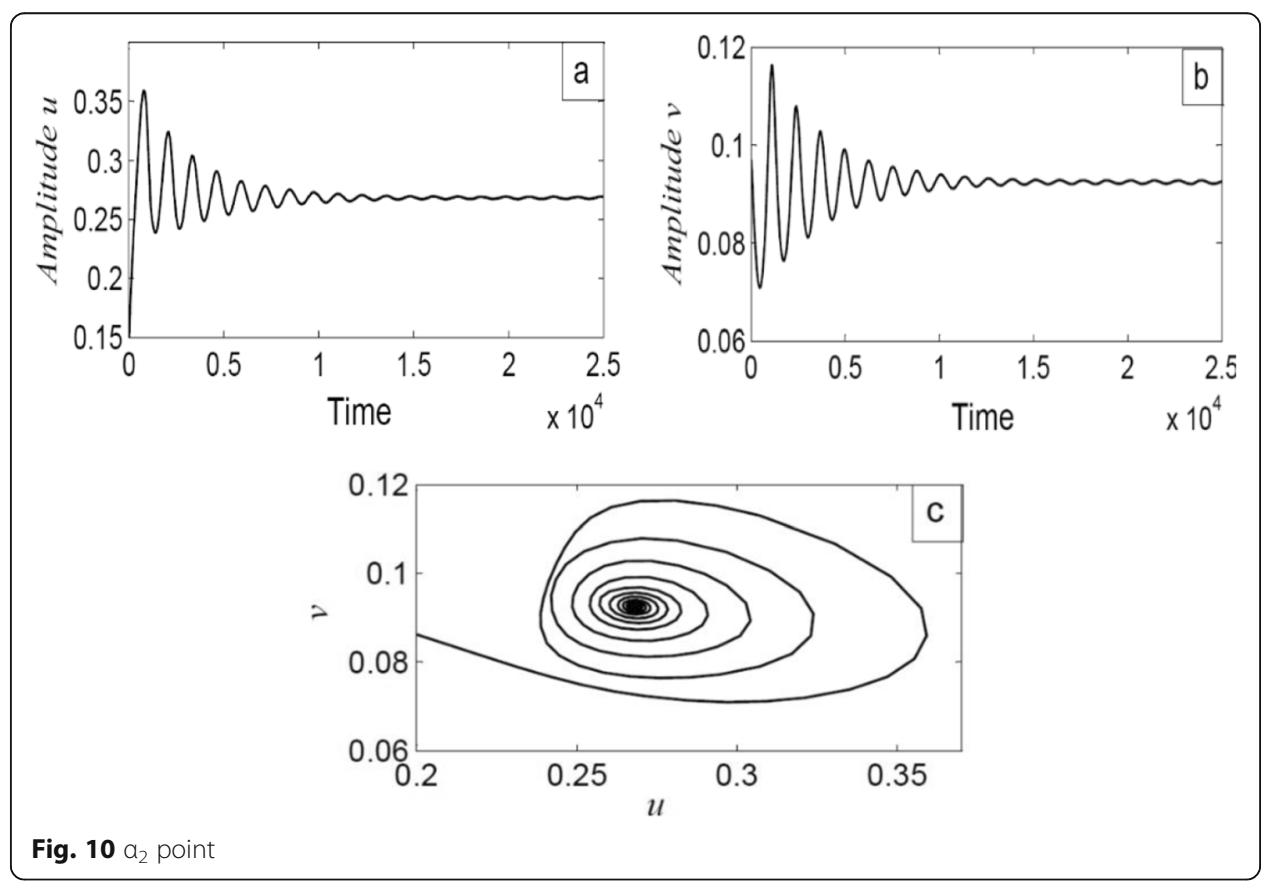




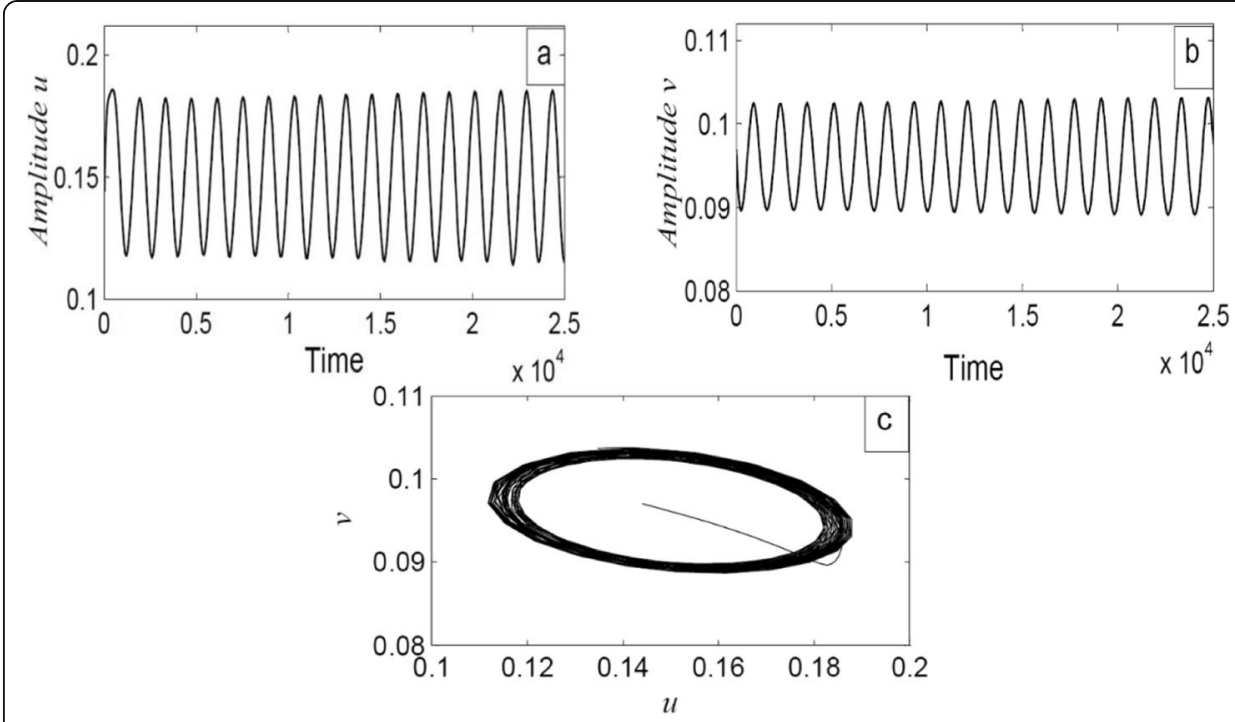

Fig. $11 a_{2}$ periodic

Region $(\gamma \leq 47.56)$ : The system is controlled by only a stable steady state where $a_{1}$ exists in the range of $[0.01,0.1691]$ (Fig. 14a) while $a_{2}$ changes in the range of $[0.575$, 0.08244] as shown in Fig. 14b.

Region $(47.56 \leq \gamma)$ : In this region, the system is dominated by stable periodic attractor, where the frequency of $a_{1}$ increases as $\gamma$ increases in the right side until it reaches the maximum at $\gamma=47.56$, where $a_{1}$ oscillates in the range of $[0.01,0.95]$ as shown in Fig. 14a while $a_{2}$ oscillates in the range of [0.025, 0.18] as shown in Fig. 14b.

\section{Effect of the natural frequency $\left(\omega_{1}\right)$}

The effect of $\omega_{1}$ as the main bifurcation parameter is indicated in Fig. 15 where the other parameter values are taken from Table 1 . There is only HB point at $\omega_{1}=3.283$, $a_{1}=0.01664$, and $a_{2}=0.5767$ as shown in Fig. 15a, b. The system behavior due to changing $\omega_{1}$ can be described as follows:

Region $\left(3.283 \leq \omega_{1}\right)$ : In this region, the system is controlled by point attractor, where $a_{1}$ changes in the range of $[0.017,0.02]$, and $a_{2}$ changes in the range of $[0.57,0.6]$ as shown in Fig. 15a, b, respectively.
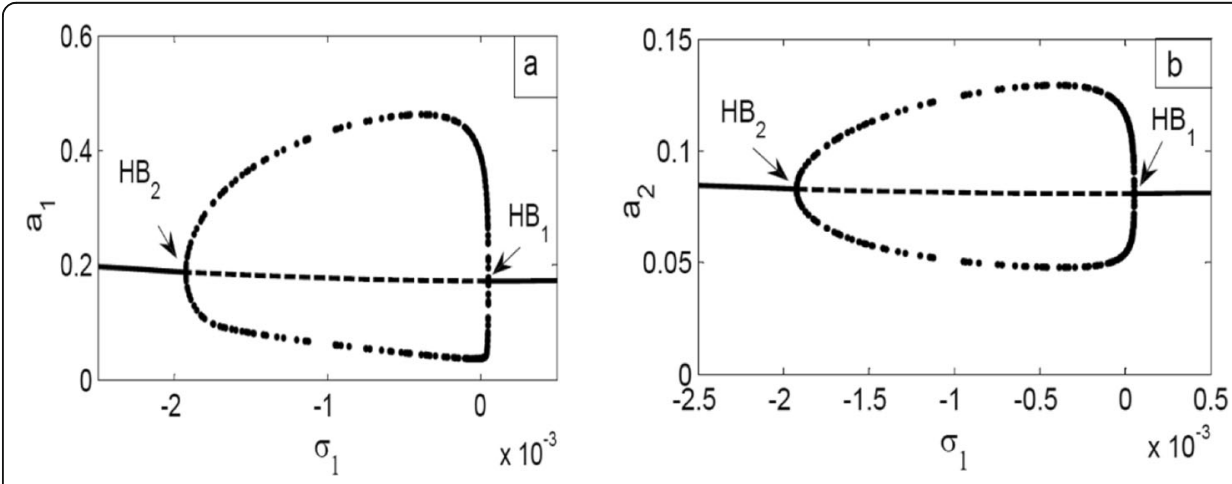

Fig. 12 Bifurcation diagram of detuning parameter $\sigma_{1}$ as the bifurcation parameter 

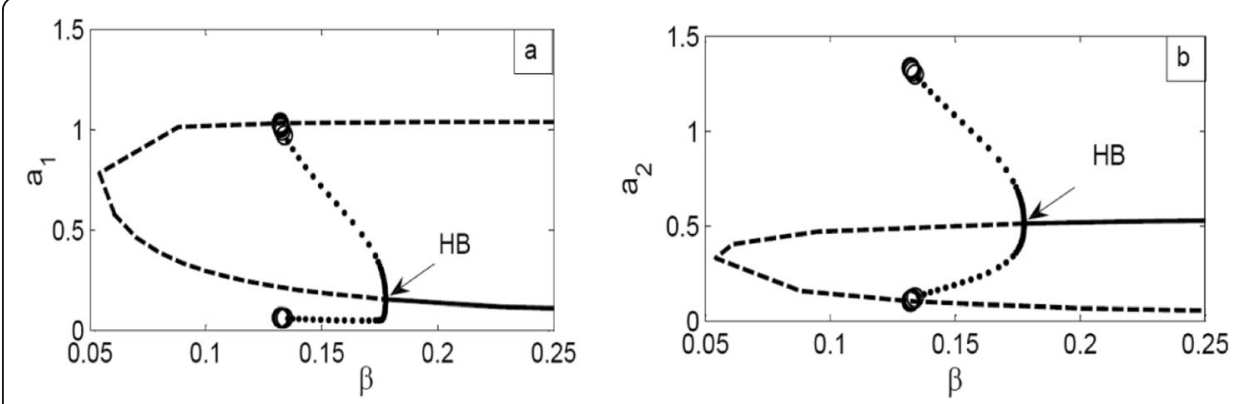

Fig. 13 Bifurcation diagram of feedback signal gain $\beta$ as the bifurcation parameter

Region $\left(2.85 \leq \omega_{1} \leq 3.283\right)$ : The system is dominated by periodic attractors where there are stable periodic attractors and unstable point attractors. Figure 15 a shows that the frequency of $a_{1}$ increases as $\omega_{1}$ decreases in this region until it reaches the maximum at $\omega_{1}=2.85$ where $a_{1}$ oscillates in the range [0.01,0.24]. Figure $15 \mathrm{~b}$ shows that the $a_{2}$ has the maximum frequency range of $[0.5,1.2]$ at $\omega_{1}=2.85$.

Region $\left(\omega_{1} \leq 2.85\right)$ : In this region, the system has unstable oscillations and unstable steady state. There is neither point nor periodic attractors as shown in Fig. 15a, b.

\section{Numerical validation}

Figure 16 shows time trace of the controlled system Eqs. (1) and (2) and the approximate modulated amplitudes of Eqs. (34)-(37) which obtained from perturbation analysis of the controlled system. From the comparison, we concluded that the predictions obtained from approximated analytical solutions using the MSP method agree well with the numerical solutions found by the RK.

Figures 17 and 18 show a good agreement between the numerical solution of the original system (1) and (2) using the RK method and approximate solution using the MSP method. Also, the results obtained in Fig. 17 agreement with the results obtained in Fig. 5. The saturation phenomenon occurs for increasing feedback signal gain $\beta$ as shown in Fig. 18.

\section{Comparison with previous work}

In [26], the active vibration controller 1:2 for suppressing the vibration of the nonlinear composite beam subjected to parametric excitation force only has been studied. In this paper, an active vibration controller 1:3 for suppressing the vibration of the nonlinear
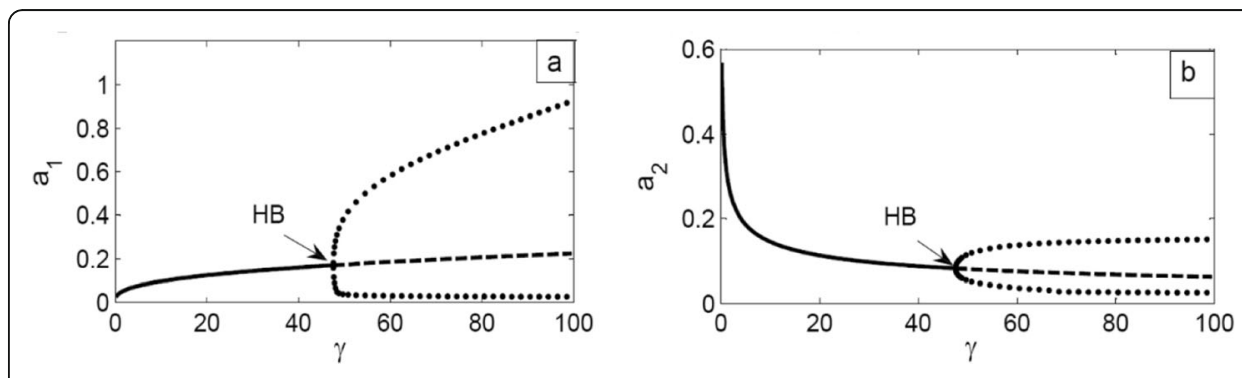

Fig. 14 Bifurcation diagram of the control signal gains $\gamma$ as the bifurcation parameter 

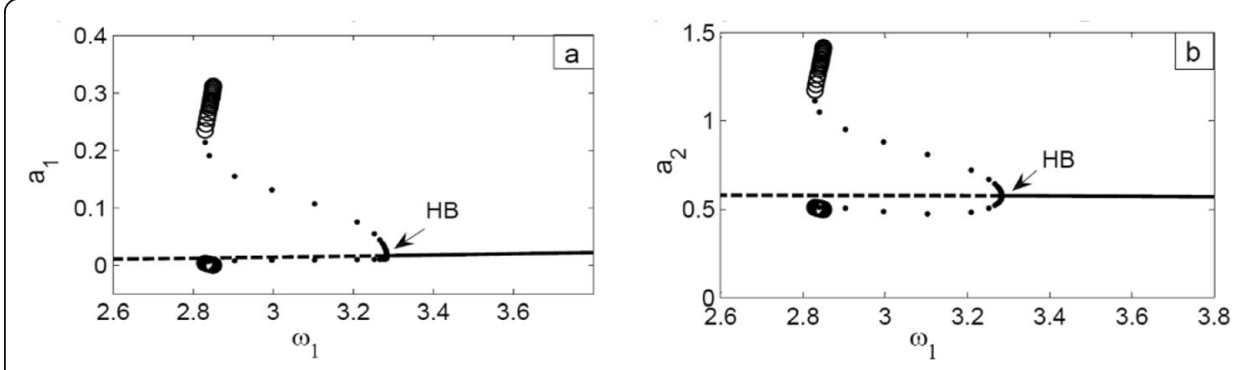

Fig. 15 Bifurcation diagram of natural frequency $\omega_{1}$ as the bifurcation parameter

composite beam subjected to parametric and external excitation forces has been studied. Bifurcation analysis is conducted using various control parameters such as natural frequency $\left(\omega_{1}\right)$, detuning parameter $\left(\sigma_{1}\right)$, feedback signal gain $(\beta)$, control signal gain $(\gamma)$, and other parameters. The dynamic behavior of the system is predicted within various ranges of bifurcation parameters. All of the stable steady state (point attractor), stable periodic attractor, unstable steady state, and unstable periodic attractors were determined efficiently using bifurcation analysis. The controller's influence on system behavior is examined numerically.

\section{Conclusions}

The main objective of the work is to control the vibration of a nonlinear composite cantilever beam with external and parametric excitation forces. We applied the MSP technique to solve those nonlinear equations. We applied Lyapunov's first method to investigate the stability of the controlled system. The corresponding FR equations have been extracted and presented graphically at different system parameters. The obtained curves were confirmed numerically applying the RK algorithm. Bifurcation analysis was conducted using many bifurcation parameters such as $f_{2}, \alpha_{2}, \sigma_{1}, \beta, \gamma$, and $\omega_{1}$. The nonlinear controller has been studied at $\Omega_{1} \cong \omega_{1}, \Omega_{2} \cong 2 \omega_{1}, \omega_{1} \cong 3 \omega_{2}$. To validate our results, the approximated analytical solution using the MSP perturbation method is compared with the numerical solution using the RK method of order four. From a previous comprehensive study, the following points are concluded:

1. The composite beam amplitude in the case of no controller at the simultaneous principal and primary parametric resonance $\Omega_{1} \cong \omega_{1}, \Omega_{2} \cong 2 \omega_{1}$ is multiplied to about 30 times of the parametric excitation amplitude $f_{2}$.
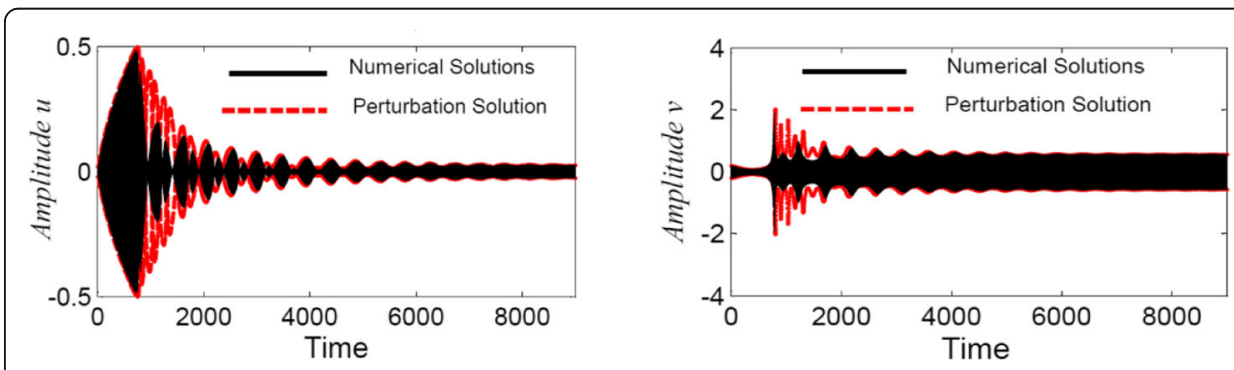

Fig. 16 Comparison between RK and MSP methods at $\Omega_{1} \cong \omega_{1}, \Omega_{2} \cong 2 \omega_{1}, \omega_{1} \cong 3 \omega_{2}$ 

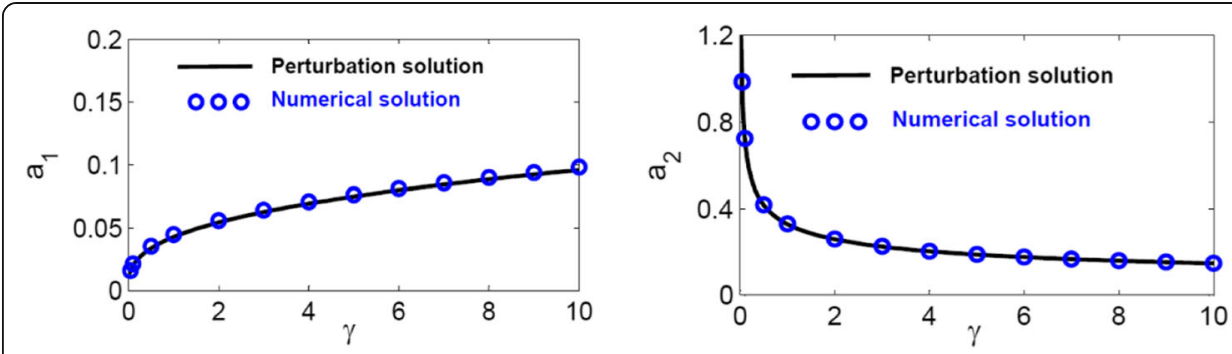

Fig. 17 Comparison between RK and MSP methods at $\Omega_{1} \cong \omega_{1}, \Omega_{2} \cong 2 \omega_{1}, \omega_{1} \cong 3 \omega_{2}$

2. The controller $1: 3$ is efficient for lowering the high steady state amplitude vibration of the composite beam.

3. Increasing the feedback signal $\beta$ obtained the better of the vibration damping effect of the composite beam.

4. For increasing the linear damping $\mu_{1}$ and natural frequency $\omega_{1}$, the efficient frequency bandwidth of the controller and the better of vibration damping the composite beam influence.

5. For decreasing the control signal $\gamma$ and excitation forces $f_{1}$ and $f_{2}$, the effective frequency bandwidth of the saturation controller can be improved and increase the vibration reduction action of the composite beam.

6. The overload controller risk decreases as the control signal $\gamma$ increases.

7. The saturation phenomenon occurs for increasing feedback signal gain $\beta$.

8. The excitation force amplitude is increased as the steady state amplitude increased and lost its stability.

9. The composite beam amplitude is inversely proportional to the damping coefficient $\mu_{1}$ and natural frequency $\omega_{1}$.

10. The validation of our results has been done, where the approximate analytical solutions are compatible with the numerical solutions.

11. The dynamic system behavior was determined at different regions of the bifurcation parameters where all stable steady state, stable periodic, unstable steady state, and periodic attractors were evaluated.

In future work, we present some suggestions for further developments. Involving higher order nonlinearities to make the mathematical model closer to the real-life of a composite cantilever beam. Also, we intend to implement another control.
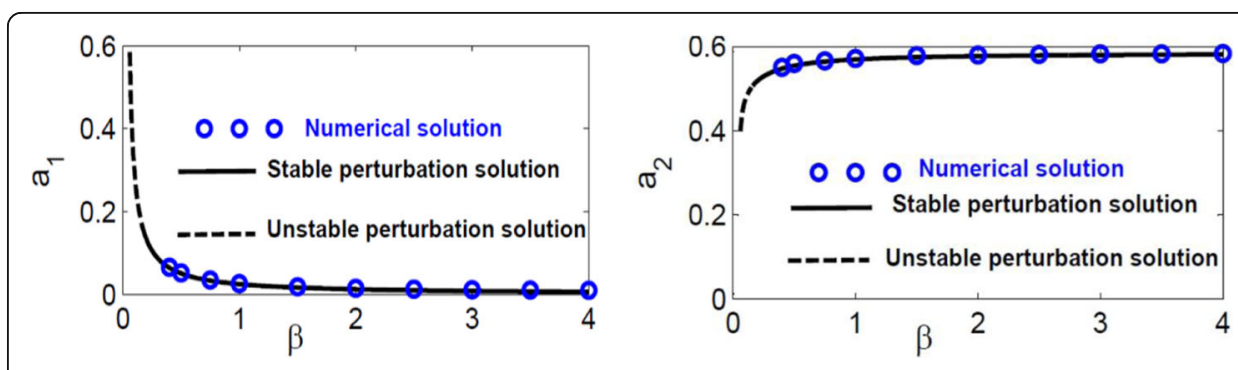

Fig. 18 Comparison between RK and MSP methods at $\Omega_{1} \cong \omega_{1}, \Omega_{2} \cong 2 \omega_{1}, \omega_{1} \cong 3 \omega_{2}$ 


\section{Acknowledgements}

The authors would like to thank the referees for their valuable comments and suggestions, which helped to improve the quality of the manuscript.

\section{Authors' contributions}

MS wrote the title, abstract, stability, graph the figures, and conclusion and fixed many language errors. AAM wrote the introduction and references. DYA wrote the mathematical analysis. IHM wrote the bifurcation analysis. SIE wrote the numerical analysis. All authors read and approved the final manuscript.

\section{Funding}

There is no sponsor.

\section{Availability of data and materials}

Data sharing not applicable to this article as no datasets were generated or analyzed during the current study.

\section{Competing interests}

The authors declare that they have no competing interests.

\section{Author details}

${ }^{1}$ Department of Engineering Mathematics, Faculty of Electronic Engineering, Menoufia University, Menouf 32952, Egypt. ${ }^{2}$ Mathematics and Statistics Department, Faculty of Science, Taif University, Taif, Kingdom of Saudi Arabia. ${ }^{3}$ Basic Engineering Sciences Department, Faculty of Engineering, Menoufia University, Shibin El-Kom, Egypt. ${ }^{4}$ Mathematics Department, Faculty of Arts and Science in Baljurashi, Al-Baha University, Al Baha, Kingdom of Saudi Arabia. ${ }^{5}$ Biomedical Engineering Department, Helwan University, Cairo, Egypt. ${ }^{6}$ Chemical Engineering Department, University of Waterloo, Waterloo, Canada. ${ }^{7}$ Department of Mathematics, Faculty of Science, Tanta University, Tanta, Egypt.

Received: 8 February 2020 Accepted: 28 July 2020

Published online: 21 September 2020

\section{References}

1. Fanson, J., Caughey, T.: Positive position feedback control for large space structures. AlAA J. 28(4), 717-724 (1990)

2. Shen, Y., Guo, W., Pao, Y.C.: Torsional vibration control of a shaft through active constrained layer damping treatments. J. Vibration Acoust. 119(4), 504-511 (1997)

3. Liu, Y., Wang, K.W.: A non-dimensional parametric study of enhanced active constrained layer damping treatments. J. Sound Vib. 223(4), 611-644 (1999)

4. Stanawy, R., Chantalakhana, D.: Active constrained layer damping of clamped-clamped plate vibrations. J. Sound Vib. 241(5), 755-777 (2001)

5. Ray, M.C., Oh, J., Baz, A.: Active constrained layer damping of thin cylindrical shells. J. Sound Vib. 240(5), 921-935 (2001)

6. Shi, Y.M., Li, Z.F., Hua, H.X., Fu, Z.F., Liu, T.X.: The modeling and vibration control of beams with active constrained layer damping. J. Sound Vib. 245(5), 785-800 (2001)

7. Sun, D., Tong, L.: Modeling and vibration control of beams with partially de-bonded active constrained layer damping patch. J. Sound Vib. 252(3), 493-507 (2002)

8. Park, C.H., Baz, A.M.: Vibration damping and control using active constrained layer damping: a survey. Shock Vibration Digest. 31(5), 355-364 (1999)

9. El-Badawy, A.A., Nayfeh, A.H.: Control of a directly excited structural dynamic model of an F-15 tail section. J. Franklin Inst. 338(2-3), 133-147 (2001)

10. Eissa, M., Sayed, M.: A comparison between passive and active control of non-linear simple pendulum Part-I. Math. Comput. Appli. 11(2), 137-149 (2006)

11. Eissa, M., Sayed, M.: A comparison between passive and active control of non-linear simple pendulum Part-II. Math. Comput. Appli. 11(2), 151-162 (2006)

12. Eissa, M., Sayed, M.: Vibration reduction of a three DOF non-linear spring pendulum. Commun. Nonlinear Sci Numerical Simulation. 13(2), 465-488 (2008)

13. El-Bassiouny, A.F.: Internal resonance of a nonlinear vibration absorber. Physica Scripta. 72(2-3), 203-211 (2005)

14. Eissa, M., EL-Serafi, S., El-Sherbiny, H., El-Ghareeb, T. H., "Comparison between passive and active control of non-linear dynamical system", Jpn. J.f Indust. App. Math., 23(2), 139-161, (2006).

15. Eissa, M., EL-Serafi, S., El-Sherbiny, H., El-Ghareeb, T. H., "On passive and active control of vibrating system", Int. J. Appl. Math., 18(4), 515-537, (2005).

16. Eissa, M., EL-Serafi, S., El-Sherbiny, H., El-Ghareeb, T. H., " $1: 4$ Internal resonance active absorber for non-linear vibrating system", Int. J. Pure App. Math., 28(1), 515-537, (2006).

17. Eissa, M., Amer, Y.A., Bauomey, H.S.: Active control of an aircraft tail subject to harmonic excitation. Acta Mechanica Sinica. 23(4), 451-462 (2007)

18. Jaensch, M., Lampérth, M.U.: Development of a multi-degree-of freedom micro positioning, vibration isolation and vibration suppression system. Smart Materials Structures. 16(2), 409-417 (2007)

19. Sadek, I.S., Kucuk, I., Adali, S.: Active open-loop control of plates with multiple piezoelectric patches via the maximum principle. Mech. Adv. Materials Structures. 21(9), 772-779 (2014)

20. Wang, X., Alici, G., Tan, X.: Modeling and inverse feedforward control for conducting polymer actuators with hysteresis. Smart Materials Structures. 23(2), 025015-025023 (2014)

21. Dong, X.J., Meng, G., Peng, J.C.: Vibration control of piezoelectric smart structures based on system identification technique: numerical simulation and experimental study. J. Sound Vib. 297(3-5), 680-693 (2006)

22. Kapuria, S., Yasin, M.Y.: Active vibration suppression of multilayered plates integrated with piezoelectric fiber reinforced composites using an efficient finite element model. J. Sound Vib. 329(16), 3247-3265 (2010) 
23. Warminski, J., Cartmell, M.P., Bochenski, M., Ivanov, I.: Analytical and experimental investigations of an autoparametric beam structure. J. Sound Vib. 315(3), 486-508 (2008)

24. Warminski, J., Bochenski, M., Jarzyna, W., Filipek, P., Augustyniak, M.: Active suppression of nonlinear composite beam vibrations by selected control algorithms. Commun. Nonlinear Sci Numerical Simulation. 16(5), 2237-2248 (2011)

25. El-Ganaini, W.A., Saeed, N.A., Eissa, M.: Positive position feedback (PPF) controller for suppression of nonlinear system vibration. Nonlinear Dynamics. 72(3), 517-537 (2013)

26. Hamed, Y.S., Amer, Y.A.: Nonlinear saturation controller for vibration supersession of a nonlinear composite beam. J. Mech.I Sci. Technol. 28(8), 2987-3002 (2014)

27. Sayed, M., Kamel, M.: Stability study and control of helicopter blade flapping vibrations. App. Math. Model. 35(6), 2820$2837(2011)$

28. Sayed, M., Kamel, M.: 1:2 and 1:3 internal resonance active absorber for non-linear vibrating system. App. Math. Model. 36(1), 310-332 (2012)

29. Wei, H., Pan, Q.X., Adetoro, O.B., Avital, E., Yuan, Y., Wen, P.H.: Dynamic large deformation analysis of a cantilever beam. Math. Comput. Simulation. 174, 183-204 (2020)

30. Chentouf, B., Wang, J.-M.: Optimal energy decay for a nonhomogeneous flexible beam with a tip mass. J. Dynamical Control Syst. 13(1), 37-53 (2007)

31. Chentouf, B.: Boundary feedback stabilization of a variant of the SCOLE model. J. Dynamical Control Syst. 9(2), 201-232 (2003)

32. Chentouf, B.: Modelling and stabilization of a nonlinear hybrid system of elasticity. App. Math. Model. 39, 621-629 (2015)

33. Bağdatlı, S.M., Öz, H.R., Özkaya, E.: Non-linear transverse vibrations and 3:1 internal resonances of a tensioned beam on multiple supports. Math. Comput. Appli. 16(1), 203-215 (2011)

34. Hegazy, U.H.: 3:1 Internal resonance of a string-beam coupled system with cubic nonlinearities. Commun. Nonlinear Sci Numerical Simulation. 15, 4219-4229 (2010)

35. Tunç, C: A note on the stability and boundedness results of solutions of certain fourth order differential equations. Appl. Math Comput. 155(3), 837-843 (2004)

36. Tunç, C.: Some remarks on the stability and boundedness of solutions of certain differential equations of fourth-order. Comput. App. Math. 26(1), 1-17 (2007)

37. Qingkai, K: A Short Course in Ordinary Differential Equations. Springer International Publishing Switzerland (2014)

38. Nayfeh, A.H.: Non-linear Interactions. Wiley-Inter-Science, New York (2000)

39. Nayfeh, A.H., Mook, D.T.: Perturbation Methods. John Wiley \& Sons, Inc. (1973)

40. Mustafa, I.H., Ibrahim, G., Elkamel, A., Elnashaie, S.S.E.H., Chen, P.: Nonlinear feedback modeling and bifurcation of the acetylcholine neurocycle and its relation to Alzheimer's and Parkinson's diseases. Chem. Eng. Sci. 64(1), 69-90 (2009)

41. Mustafa, I.H., Elkamel, A., Ibrahim, G., Chen, P., Elnashaie, S.S.E.H.: Effect of choline and acetate substrates on bifurcation and chaotic behavior of acetylcholine neurocycle and Alzheimer's and Parkinson's diseases. Chem. Eng. Sci. 64(9), 2096$2112(2009)$

42. Mustafa, I.H., Elkamel, A., Lohi, A., Chen, P., Elnashaie, S.S.E.H., Ibrahim, G.: Application of continuation method and bifurcation for acetylcholine neurocycle considering partial dissociation of acetic acid. Comput. Chem. Eng. 46, 78-93 (2012)

43. Mustafa, I.H., Elkamel, A., Chen, P., Ibrahim, G., Elnashaie, S.S.E.H.: Effect of cholineacetyltransferase activity and choline recycle ratio on modelling, bifurcation and chaotic behavior of acetylcholine neurocycle and their relation to Alzheimer's and Parkinson's diseases. Chem. Eng. Sci. 68(1), 19-35 (2012)

44. Mustafa, I.H.: Two-parameter continuation and bifurcation strategies for oscillatory behavior elimination from a zymomonas mobilis fermentation system. Chem. Eng. Technol. 38(8), 1362-1370 (2015)

\section{Publisher's Note}

Springer Nature remains neutral with regard to jurisdictional claims in published maps and institutional affiliations.

\section{Submit your manuscript to a SpringerOpen ${ }^{\circ}$ journal and benefit from:}

- Convenient online submission

- Rigorous peer review

Open access: articles freely available online

- High visibility within the field

- Retaining the copyright to your article

Submit your next manuscript at $\boldsymbol{\nabla}$ springeropen.com 